\title{
The Clinical, Microbiological, and Immunological Effects of Probiotic Supplementation on Prevention and Treatment of Periodontal Diseases: A Systematic Review and Meta-Analysis
}

\author{
Zohre Gheisary ${ }^{1, \dagger}{ }^{,}$Razi Mahmood ${ }^{1,+}\left(\mathbb{0}\right.$, Aparna Harri shivanantham ${ }^{1}\left(\mathbb{0}\right.$, Juxin Liu ${ }^{2}$, Jessica R. L. Lieffers ${ }^{3}(\mathbb{D}$, \\ Petros Papagerakis ${ }^{4}$ and Silvana Papagerakis ${ }^{1, *}$
}

check for updates

Citation: Gheisary, Z.; Mahmood, R.; Harri shivanantham, A.; Liu, J.; Lieffers, J.R.L.; Papagerakis, P.; Papagerakis, S. The Clinical,

Microbiological, and Immunological Effects of Probiotic Supplementation on Prevention and Treatment of Periodontal Diseases: A Systematic Review and Meta-Analysis. Nutrients 2022, 14, 1036. https://doi.org/ $10.3390 /$ nu14051036

Academic Editors: Kirstin Vach and Johan Peter Woelber

Received: 12 January 2022

Accepted: 23 February 2022

Published: 28 February 2022

Publisher's Note: MDPI stays neutral with regard to jurisdictional claims in published maps and institutional affiliations.

Copyright: (C) 2022 by the authors. Licensee MDPI, Basel, Switzerland. This article is an open access article distributed under the terms and conditions of the Creative Commons Attribution (CC BY) license (https:// creativecommons.org/licenses/by/ $4.0 /)$.
1 Laboratory of Oral, Head and Neck Cancer-Personalized Diagnostics and Therapeutics, College of Medicine, University of Saskatchewan, 107 Wiggins Road, Saskatoon, SK S7N 5E5, Canada; zog389@usask.ca (Z.G.); razi.mahmood@usask.ca (R.M.); eti908@mail.usask.ca (A.H.s.)

2 Department of Mathematics and Statistics, College of Arts and Science, University of Saskatchewan, 106 Wiggins Road, Saskatoon, SK S7N 5E6, Canada; jul086@mail.usask.ca

3 College of Pharmacy and Nutrition, University of Saskatchewan, 107 Wiggins Road, Saskatoon, SK S7N 5E5, Canada; jessica.lieffers@usask.ca

4 Laboratory of Precision Oral Health and Chronobiology, College of Dentistry, University of Saskatchewan, 107 Wiggins Road, Saskatoon, SK S7N 5E5, Canada; petros.papagerakis@usask.ca

* Correspondence: silvana.papagerakis@usask.ca; Tel.: +1-3069661960

+ These authors contributed equally to this work.

Abstract: (1) Background: Periodontal diseases are a global health concern. They are multi-stage progressive inflammatory diseases triggered by the inflammation of the gums in response to periodontopathogens and may lead to the destruction of tooth-supporting structures, tooth loss, and systemic health problems. This systematic review and meta-analysis evaluated the effects of probiotic supplementation on the prevention and treatment of periodontal disease based on the assessment of clinical, microbiological, and immunological outcomes. (2) Methods: This study was registered under PROSPERO (CRD42021249120). Six databases were searched: PubMed, MEDLINE, EMBASE, CINAHL, Web of Science, and Dentistry and Oral Science Source. The meta-analysis assessed the effects of probiotic supplementation on the prevention and treatment of periodontal diseases and reported them using Hedge's g standardized mean difference (SMD). (3) Results: Of the 1883 articles initially identified, 64 randomized clinical trials were included in this study. The results of this meta-analysis indicated statistically significant improvements after probiotic supplementation in the majority of the clinical outcomes in periodontal disease patients, including the plaque index $(\mathrm{SMD}=0.557,95 \% \mathrm{CI}: 0.228,0.885)$, gingival index, SMD $=0.920,95 \% \mathrm{CI}: 0.426,1.414)$, probing pocket depth $(\mathrm{SMD}=0.578,95 \% \mathrm{CI}: 0.365,0.790)$, clinical attachment level $(\mathrm{SMD}=0.413,95 \% \mathrm{CI}$ : $0.262,0.563)$, bleeding on probing (SMD $=0.841,95 \% \mathrm{CI}: 0.479,1.20)$, gingival crevicular fluid volume (SMD $=0.568,95 \%$ CI: 0.235, 0.902), reduction in the subgingival periodontopathogen count of P. gingivalis (SMD $=0.402,95 \%$ CI: 0.120, 0.685), F. nucleatum (SMD $=0.392,95 \%$ CI: $0.127,0.658$ ), and T. forsythia (SMD $=0.341,95 \%$ CI: 0.050, 0.633), and immunological markers MMP-8 (SMD = 0.819, 95\% CI: $0.417,1.221)$ and IL-6 (SMD $=0.361,95 \%$ CI: 0.079, 0.644). (4) Conclusions: The results of this study suggest that probiotic supplementation improves clinical parameters, and reduces the periodontopathogen load and pro-inflammatory markers in periodontal disease patients. However, we were unable to assess the preventive role of probiotic supplementation due to the paucity of studies. Further clinical studies are needed to determine the efficacy of probiotic supplementation in the prevention of periodontal diseases.

Keywords: probiotic; periodontal disease; gingivitis; periodontitis; oral health; clinical parameters; prevention; therapeutics 


\section{Introduction}

Periodontal disease is a growing public health concern, affecting approximately 750 million individuals worldwide [1]. The burden of this disease is expected to continue to grow as the global population ages [2,3]. Periodontal disease is preventable and reversible in its early stages; however, it can progress to chronic, irreversible states with significant destruction of the tooth-supporting tissues [4]. The cause of periodontal disease is multifactorial with modifiable risk factors, including smoking, unhealthy diet (e.g., a western diet with high sugars and saturated fats), poor oral hygiene, hormonal changes, stress, various medications, and poorly managed comorbidities (e.g., type 2 diabetes), while non-modifiable risk factors include age, sex, and genetics [5]. Periodontal disease, when left untreated, can have local and/or systemic consequences, leading to poor oral and systemic health and quality of life $[5,6]$. The underlying link of periodontal disease with other chronic systemic diseases likely results from the dissemination of periodontopathogens into the bloodstream, endotoxin release, and the associated imbalanced inflammatory response to periodontopathogens $[7,8]$.

Periodontal disease is an inflammatory progressive multi-stage disease of the periodontium (which includes the gingiva, periodontal ligament, alveolar bone, and cementum); this disease is triggered in response to periodontopathogens in the biofilm of the dental plaque on tooth surfaces located near the gingiva (Figure 1) [9-11]. The first stage and mildest form of periodontal disease is known as gingivitis [4]. Gingivitis is a reversible condition, and, if untreated, may progress to periodontitis, which is the advanced stage of periodontal disease $[4,12,13]$. Gingivitis is characterized by redness, swelling, mild irritation and inflammation of the gingival tissue, and mild bleeding on brushing or flossing, while periodontitis is characterized by deep inflammation and loss of alveolar bone and connective tissue between the gingiva and tooth root $[9,14]$. The progression of periodontal disease is associated with dynamic shifts in the subgingival bacterial counts and composition in the periodontal pocket [15-18].

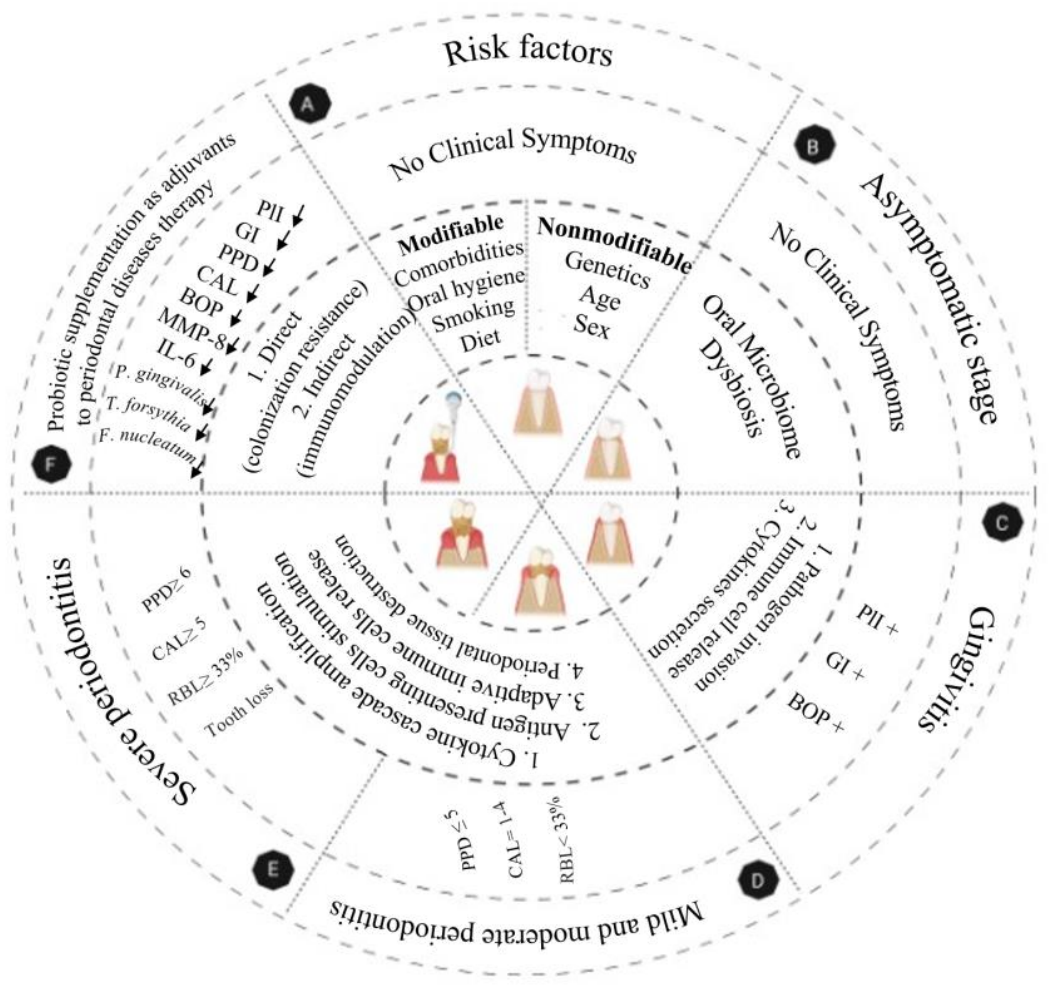

Figure 1. Etiology and pathogenesis of periodontal diseases. Periodontal disease is initiated by disrupting the commensal oral microbiome-host homeostasis. (A). Both modifiable and nonmodifiable risk factors impact the oral microbiome composition and disrupt homeostasis between the host 
and microbiome. Modifiable risk factors include diet, smoking, oral hygiene, and comorbidities (such as type 2 diabetes), while genetics, age, and sex are nonmodifiable risk factors. (B). Disrupted homeostasis provides appropriate conditions for the growth of periodontopathogens and biofilm formation on the tooth surfaces extending sub-gingivally. There are no clinical symptoms in this stage. (C). These bacteria penetrate and grow in the gingival epithelium. Host-bacteria interactions cause a chemotactic gradient that attracts innate immune cells, including neutrophils, macrophages, and NK cells, to the affected sites. In addition, the outgrowth of bacteria progressively destroys the tissue and provides enough nutrients for more pathogen growth, followed by increased activity of innate immune cells and the secretion of pro-inflammatory cytokines, including IL-1, IL-8, and TNF. Early clinical symptoms in this stage are redness, swelling, mild inflammation, and bleeding of the gingiva, which are diagnosed by measuring the PII, GI, and BOP. (D). Then, Antigen-Presenting Cells (APC), including dendritic cells, present bacterial antigens to lymphocytes and trigger adaptive immune system activity and antibody and cell-mediated immune responses, resulting in a pro-inflammatory response with high expression of IL-4, 6, 8, 10, 12, TGF- $\beta$, and IFN- $\gamma$. (E). High levels of these inflammatory mediators stimulate more inflammatory mediators, causing periodontal tissue destruction and leading to the loss of the gingival attachment to the tooth, and causing deep pockets around the teeth that provide appropriate conditions for the growth and colonization of other anaerobic periodontopathogens. Untreated, these pathophysiological changes can lead to alveolar bone resorption and, ultimately, tooth loss in the most advanced stage of the disease. (F). Probiotics may have therapeutic benefits in periodontal disease treatment when used as an adjuvant to standard periodontal care. Various mechanisms of action have been considered for the role of probiotics in periodontal disease improvement. Probiotics interact directly with periodontopathogens through colonization resistance, which includes competition for binding sites and nutrients, and the production of antibacterial agents inhibiting pathogen growth. Probiotics can play a role in periodontal disease improvement indirectly via the modulation of innate and adaptive immunity and through the gut-oral microbiome axis. PlI, Plaque Index; GI, Gingival Index; BOP, Bleeding on Probing; IL, Interleukin; TGF- $\beta$, Tumor Growth Factor- $\beta$; and IFN- $\gamma$, Interferon- $\gamma$.

\subsection{Etiology of Periodontal Disease}

The primary etiology of periodontal disease is an imbalanced subgingival microbiome population developing progressively over time due to an increasing relative abundance of periodontal disease-associated bacteria and a corresponding decrease in health-associated bacteria, leading to the disruption of the microbiota-host homeostasis [16,19-21]. This gradual phenomenon begins with the early adherence, growth, and colonization of Gramnegative and Gram-positive bacteria on the tooth surface extending sub-gingivally [22]. This provides appropriate conditions for the growth of and colonization by other anaerobic Gram-negative orange and red-complex bacteria [20]. The orange complex consists of Prevotella intermedia, Parvimonas micra, and Fusobacterium nucleatum, while Porphyromonas gingivalis, Tanerella forsythia, and Treponema denticola are components of the red complex [20]. These bacteria are highly pathogenic and have the ability to release bacterial collagenases and other proteases, leading to the stimulation of the pro-inflammatory response and periodontal tissue damage $[9,10,20]$ (Figure 1).

\subsection{Periodontal Disease Assessment, Diagnosis, and Therapy}

The clinical assessment of periodontal disease includes the evaluation of patientreported outcomes (i.e., bleeding while brushing or flossing, receding gums, halitosis, sensitive teeth, pain during mastication, and loose teeth) [4] and visual assessment of the distance between the base of the periodontal pocket and gingival margin or cementoenamel junction, the amount of plaque in the gingival margin on the tooth surface, and gingival bleeding $[9,23]$. All of these factors are measured by standard procedures and defined as specific indexes, including: the plaque index (PII), gingival index (GI), periodontal pocket 
depth (PPD), clinical attachment level (CAL), bleeding on probing (BOP), gingival recession (REC), and gingival crevicular fluid (GCF) volume [23-27].

The clinical diagnosis of periodontal diseases is based on the periodontal exam, radiography, and patient's oral/dental and medical history [28]. The American Academy of Periodontology (AAP) classifies periodontitis into four stages based on severity, complexity, and extent (stage I, II, III, and IV) and three grades based on the evidence of the disease's progression and its rate (slow, moderate, and rapid) [29,30].

Periodontal therapy consists of the removal of the supra- and subgingival plaque from tooth surfaces using Scaling and Root Planing (SRP) [31,32]. To improve disease outcomes, SRP can be incorporated into surgical procedures or adjunctive antibiotics can be administered [3,31-33]. However, antibiotics may cause adverse side effects, or can be contraindicated in some situations; therefore, there is a need for alternative approaches [14].

\subsection{Periodontal Diseases and Probiotics}

Increasing attention has been devoted to probiotic supplementation as a therapeutic adjuvant/alternative to improve oral health [34]. Probiotics are live organisms (usually bacteria) administered to provide health benefits in the prevention or clinical management of different diseases $[35,36]$. Probiotics have been traditionally accepted in the medical field as an adjuvant treatment of gastrointestinal disorders [37]. Moreover, probiotics are recommended to patients who take antibiotics for the prevention and treatment of Antibiotics-Associated Diarrhea (AAD) [38]. They have also been considered in the clinical management of other conditions, including respiratory tract infections [39]. Furthermore, probiotics may have a therapeutic benefit in dental caries prevention by decreasing the number of cariogenic bacteria, such as Streptococcus mutans [40]. Probiotics may function through various mechanisms, including the production of antimicrobial metabolites, immunomodulation, mucosal barrier enhancement, and microbial flora shift through competition for cell adhesion with pathogenic strains [34,41]. The use of probiotics for the clinical management of periodontal diseases is an active area of research. There have been conflicting results based on several individual studies assessing the effects of probiotics on gingival inflammation [42,43]. Furthermore, previously published systematic reviews have had limited findings with conflicting results when examining the clinical efficacy of probiotics on periodontal diseases. For instance, Akram et al. showed no improvement in $\mathrm{PII}$ and GI in patients with gingivitis after probiotic use, while other reviews concluded that probiotics could improve PII and GI [44-46]. A previous systematic review was unable to assess the immunological benefits of probiotic supplementation due to limited studies; however, the included individual studies indicated an immunomodulatory effect of probiotics [47]. Microbiological findings suggested that probiotic supplementation reduced periodontopathogens in subgingival plaque samples [47]; however, there is conflicting evidence in the literature [48].

The purpose of our systematic review and meta-analysis was to combine results from randomized clinical trials involving adults with periodontal diseases or healthy volunteers receiving probiotic supplementation (control groups did not receive probiotic supplementation) to assess the effects on the clinical, microbiological, and immunological outcomes related to periodontal disease prevention and management.

\section{Materials and Methods}

\subsection{Eligibility Criteria}

Studies that were eligible for inclusion in this review satisfied the following criteria: 1. Randomized controlled trials with adults aged 18 years or older clinically diagnosed with either periodontal disease or healthy adults (without periodontal disease); 2 . The study design consisted of intervention groups that received probiotics in any form (i.e., lozenge, capsule, tablet, powder, probiotic drink, probiotic-fortified food, toothpaste, mouthwash, spray, or subgingival delivery) and control groups (without probiotic, with a placebo, or with antibiotics); 3 . Studies assessing any of the following: clinical, microbiological, or 
immunological outcomes. Clinical outcomes included: plaque index (PII), gingival index (GI), probing pocket depth (PPD), clinical attachment level (CAL), bleeding on probing (BOP), gingival recession (REC), and gingival crevicular fluid (GCF) volume. Microbiological outcomes included the subgingival count of periodontopathogens, including: Porphyromonas gingivalis (P. gingivalis), Fusobacterium nucleatum (F. nucleatum), Tannerella forsythia (T. forsythia), Prevotella intermedia (P. intermedia), and Aggregatibacter actinomycetemcomitans (A. actinomycetemcomitans), and of commensal oral microbiota, such as Streptococcus mutans (S. mutans) and Lactobacillus species. Immunological outcomes included the GCF levels of matrix metalloproteinase-8 (MMP-8), interleukin-6 (IL-6), interleukin-1 $\beta$ (IL-1 $\beta$ ), interleukin-8 (IL-8), interleukin-10 (IL-10), and tumor necrosis factor- $\alpha$ (TNF- $\alpha$ ).

English language, peer-reviewed studies published since 2000, which were either open access or accessible to the researchers (via the University of Saskatchewan Library, inter-library loan, or through Google scholar), were included. Detailed information about the inclusion and exclusion criteria are available in Supplemental Table S1.

\subsection{Information Sources, Search Strategy, and Study Selection}

Six databases were searched without restrictions, including PubMed, MEDLINE, EMBASE, CINAHL, Web of Science, and Dentistry and Oral Science Source. A combination of keywords and $\mathrm{MeSH}$ terms related to the following search domains were used: 1 . Periodontal diseases; 2. Clinical, microbiological, and immunological outcomes; 3. Probiotics. These three domains were combined with the "AND" operator. Details of the search strategy are included in the Supplementary Materials (Supplemental Table S2).

This systematic review and meta-analysis was registered with the International Prospective Register of Systematic Reviews (PROSPERO) (registration number: CRD42021249120).

After calibration, two screeners (ZG and AH) independently conducted dual screening (title and abstract, and full-text), and if consensus was not reached, a third author (RM) provided a tie-breaker vote.

\subsection{Data Items and Collection Process}

Two authors ( $\mathrm{ZG}$ and $\mathrm{AH}$ ) independently conducted data extraction, then compared the extracted data, and, in case of disagreement, they referred to the publication. The researchers used a Microsoft Excel (Microsoft Inc., Redmond, WA, USA) spreadsheet to record data pertaining to the study design, sample size, age of participants, health status, periodontal disease stage, dose and probiotic strain, treatment and follow-up durations, oral hygiene instructions, mode of probiotic delivery, clinical measurements (PII, GI, PPD, CAL, BOP, GCF, and REC), oral bacterial count (P. gingivalis, F. nucleatum, T. forsythia, P. intermedia, A. actinomycetemcomitans, S. mutans, and Lactobacillus species), immunological outcomes (MMP-8, IL-6, IL-1 $\beta$, IL-8, IL-10, and TNF- $\alpha$ ), and key findings of each study.

\subsection{Risk of Bias within Studies}

The risk of bias within studies was assessed independently by two authors (ZG and $\mathrm{AH})$ using the Cochrane risk-of-bias assessment tool version 2 designed for randomized trials [49]. This tool evaluates within-study bias by assessing the randomization process, deviations from intended interventions, missing outcome data, measurement of the outcome, and selection of the reported result. Studies were categorized as follows: low risk, some concerns, or high risk of bias.

\subsection{Summary Measures and Synthesis of Results}

Comprehensive meta-analysis version 3.3070 (Biostat Inc., Englewood, NJ, USA) was used for the meta-analysis. The meta-analysis was conducted using the mean and standard deviations from the studies at baseline until the immediate follow-up after the end of probiotic treatment. Hedge's g standardized mean difference (SMD) was calculated with a 95\% confidence interval (CI) for each individual study and pooled in the meta-analysis using random-effects models. SMD can be interpreted as follows: a value of 0 indicates 
that there is no statistically significant difference in the effect of treatment compared to the control; values greater or less than 0 indicate a difference in effect. The strength of the effect can be roughly interpreted as small (SMD =0.2), medium (SMD =0.5), and large $(\mathrm{SMD}=0.8)$ [50]. Heterogeneity was assessed using $\mathrm{I}^{2}$, which can be interpreted as low $(25 \%)$, moderate $(50 \%)$, or high $(75 \%)$ [51].

The meta-analysis used the following steps: 1 . Pooled random-effects models examining the effect of probiotic supplementation on clinical, microbiological, and immunological outcomes in periodontal disease patients compared to controls (without probiotics); 2. Pooled random-effects models examining the potential preventive effect of probiotic use on clinical, microbiological, and immunological outcomes in healthy individuals (without periodontal diseases) compared to controls (without probiotics); 3. Subgroup analysis (see Section 2.7). The results of the meta-analysis were reported as the SMD, 95\% CI, heterogeneity score $\left(\mathrm{I}^{2}\right)$, and $p$-value.

Studies lacking data on the mean, standard deviation, and sample size for treatment and/or control were excluded from the meta-analysis, unless these values could be calculated.

\subsection{Risk of Bias across Studies}

Publication bias and small study effects were evaluated using visual inspection of funnel plots and Egger's regression test. If publication bias/small study effects were detected, we used Duval and Tweedie's trim and fill methodology to correct for funnel plot asymmetry [52].

\subsection{Additional Analysis (Subgroup Analysis and Investigation of Heterogeneity)}

The following subgroup analyses were conducted: 1 . Type of periodontal disease (gingivitis, periodontitis); 2. Disease severity; 3. Probiotic treatment duration; 4. Mode of probiotic delivery; 5. Type of probiotic strain; 6. Type of lactobacillus species; and 7. Oral hygiene instructions (yes/no). In addition, subgroup analyses assessed the effects of probiotics compared to a control group receiving antibiotics. Disease severity was categorized as moderate $(\mathrm{PPD}=4-6 \mathrm{~mm}$ ) or deep (PPD $>6 \mathrm{~mm}$ ) periodontal pockets. The treatment duration was categorized as follows: 1 . Up to one month; 2 . More than one month to two months; or 3. More than two months. The mode of probiotic delivery was categorized as follows: 1. Oral (i.e., toothpaste, mouthwash, sachet applied orally, mouth spray, or oil drops); 2. Oral and ingestion (i.e., lozenges, tablets, or chewing gum); 3. Ingestion (i.e., sachet dissolved in water to drink, capsules, yogurt, or fermented milk/Yakult); or 4. Local application (i.e., subgingival delivery as a paste or gel). The type of probiotic strain was classified as follows: 1. Lactobacillus (Lactobacillus species only); 2. Mixed (Lactobacillus species and other bacterial species); and 3. Other (not lactobacillus species). The type of Lactobacillus was classified as follows: 1. Lactobacillus Reuteri; and 2. Other Lactobacillus spp. (any Lactobacillus species other than L. Reuteri). The oral hygiene instructions were categorized as follows: 1. Yes (when providing specific oral hygiene instructions); or 2. No (when asking participants to maintain their regular oral hygiene habits).

\section{Results}

\subsection{Study Selection}

A total of 1883 publications were identified through the electronic search of the six databases. After removing duplicates, two reviewers conducted title and abstract screening on 994 publications, and 890 were excluded. In the next step, 104 publications underwent full-text screening. Finally, 64 publications remained for the systematic review and 47 were included in the meta-analysis (17 studies were removed due to insufficient/incompatible data for meta-analysis) (Figure 2). 


\section{Identification of studies via databases and registers}

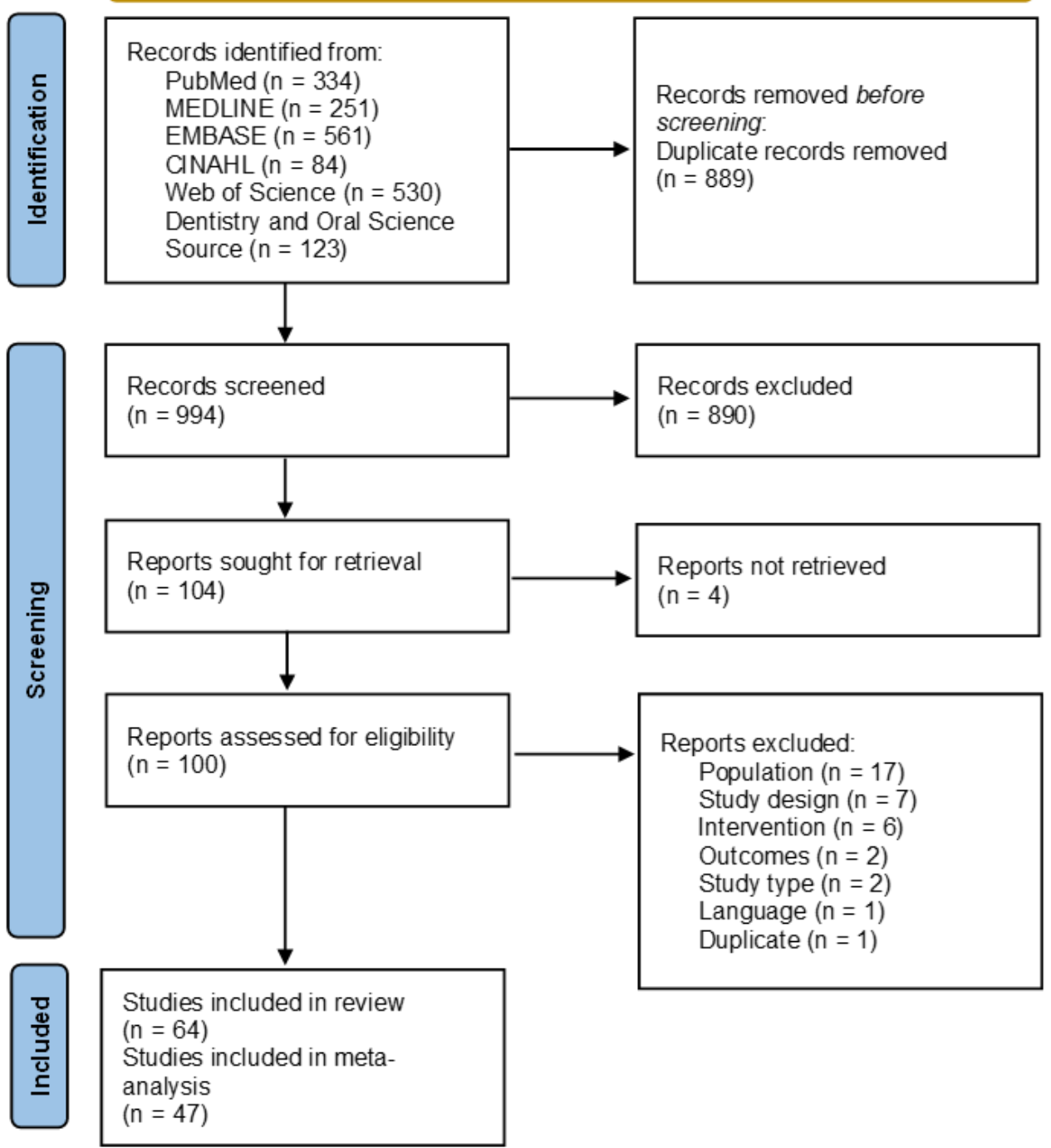

Figure 2. Preferred Reporting Items for Systematic Reviews and Meta-Analyses (PRISMA) flow diagram detailing the study selection.

\subsection{Study Characteristics}

There was a total of 64 studies eligible for the systematic review and 47 studies for the meta-analysis. Table 1 presents the detailed individual study characteristics including: periodontal disease status, sample size, probiotic strain, treatment duration and immediate follow-up, mode of probiotic delivery, other treatments, oral hygiene instructions, outcomes investigated, and key findings. The probiotic treatment duration varied from one day to four months. The sample sizes varied from 10 to 120 individuals. The most common probiotic formulation was composed of Lactobacillus reuteri. The majority of studies had periodontitis patients. 
Table 1. Characteristics of the studies included in the systematic review and meta-analysis.

\begin{tabular}{|c|c|c|c|c|c|c|c|c|c|c|}
\hline \multirow{3}{*}{$\begin{array}{l}\text { Author, Year, } \\
\text { Country }\end{array}$} & \multicolumn{3}{|c|}{ Study Sample Characteristics } & \multirow{3}{*}{$\begin{array}{l}\text { Probiotic } \\
\text { Formulation }\end{array}$} & \multirow{3}{*}{$\begin{array}{l}\text { Treatment } \\
\text { Duration/ } \\
\text { Immediate } \\
\text { Follow-Up }\end{array}$} & \multirow{3}{*}{$\begin{array}{l}\text { Mode of } \\
\text { Probiotic } \\
\text { Delivery }\end{array}$} & \multirow{3}{*}{ Other Treatments } & \multirow{3}{*}{$\begin{array}{l}\text { Oral Hygiene } \\
\text { Instructions }\end{array}$} & \multirow{3}{*}{ Outcomes Investigated } & \multirow{3}{*}{ Key Findings } \\
\hline & \multirow{2}{*}{ Disease Status } & \multicolumn{2}{|c|}{ Sample Size } & & & & & & & \\
\hline & & Probiotic & Control & & & & & & & \\
\hline $\begin{array}{l}\text { Alkaya, } 2016 \text { [53] } \\
\text { Turkey }\end{array}$ & Gingivitis & 20 & 20 & $\begin{array}{l}\text { B. megaterium, } B \text {. } \\
\text { pumulus, B. subtilis }\end{array}$ & $\begin{array}{l}8 \text { weeks/ } \\
8 \text { weeks }\end{array}$ & $\begin{array}{c}\text { Toothpaste, } \\
\text { mouth rinse, } \\
\text { and toothbrush }\end{array}$ & $\begin{array}{c}\text { Supragingival } \\
\text { scaling and/or oral } \\
\text { prophylaxis }\end{array}$ & Yes & $\mathrm{PII} *, \mathrm{GI} *, \mathrm{PPD}^{*}, \mathrm{BOP} *$ & $\begin{array}{l}\text { No statistically significant } \\
\text { difference attributed to probiotic } \\
\text { use in gingivitis patients. }\end{array}$ \\
\hline $\begin{array}{l}\text { Alshareef, } 2020 \\
\text { [54] } \\
\text { Saudi Arabia }\end{array}$ & Periodontitis & 15 & 10 & $\begin{array}{l}\text { B. bifidum, } \\
\text { L. acidophilus, L. casei, } \\
\text { L. rhamnosus, } \\
\text { L. salivarius }\end{array}$ & $\begin{array}{l}30 \text { days/ } \\
30 \text { days }\end{array}$ & Lozenge & SRP & Yes & $\begin{array}{l}\text { PII *, CAL *, PPD *, GBI }{ }^{+}, \\
\text {GCF *, MMP-8 }\end{array}$ & $\begin{array}{c}\text { Statistically significant } \\
\text { improvement in GBI and greater } \\
\text { improvement in GCF with } \\
\text { probiotic use. }\end{array}$ \\
\hline $\begin{array}{l}\text { Bollero, } 2017 \text { [56] } \\
\text { Italy }\end{array}$ & Gingivitis & 19 & 21 & $\begin{array}{l}\text { B. animalis, B. bifidum, } \\
\text { L. acidophilus, } \\
\text { L. delbrueckii, } \\
\text { L. plantarum, L. reuteri, } \\
\text { L. lactis, S. thermophilus }\end{array}$ & $\begin{array}{l}1 \text { week/ } \\
1 \text { week }\end{array}$ & Mouthwash & None & Not mentioned & $\mathrm{BOP}^{+}, \mathrm{PCR}^{+}$ & $\begin{array}{l}\text { Probiotic mouthwash may serve } \\
\text { as an additional prophylactic to } \\
\text { standard oral hygiene } \\
\text { procedures. }\end{array}$ \\
\hline $\begin{array}{l}\text { Boyeena, } 2019[57] \\
\text { India }\end{array}$ & Periodontitis & 10 & 10 & $\begin{array}{l}\text { B. bifidum, B. longum, } \\
\text { L. acidophilus, } \\
\text { L. rhamnosus }\end{array}$ & $\begin{array}{l}\text { Once/ } \\
45 \text { days }\end{array}$ & Paste & $\begin{array}{l}\text { 1) SRP + tetracycline } \\
\text { fibers } \\
\text { 2) SRP + tetracycline } \\
\text { fibers + Probiotic } \\
\end{array}$ & Yes & $\begin{array}{l}\mathrm{PII}^{*}, \mathrm{PPD}^{+}, \mathrm{SBI}^{+} \text {, total } \\
\text { bacteria * }\end{array}$ & $\begin{array}{l}\text { Probiotic and tetracycline may act } \\
\text { synergistically in the treatment of } \\
\text { periodontitis. }\end{array}$ \\
\hline $\begin{array}{l}\text { Chandra, } 2016 \text { [58] } \\
\text { India }\end{array}$ & Periodontitis & 28 & 27 & S. boulardii & $\begin{array}{l}\text { Once/ } \\
1 \text { week }\end{array}$ & Paste & SRP & Yes & $\mathrm{PI}, \mathrm{MGI}^{*}, \mathrm{CAL}^{+}, \mathrm{PPD}^{+}$ & $\begin{array}{l}\text { S. boulardii and SRP significantly } \\
\text { improved periodontal disease } \\
\text { parameters compared to SRP } \\
\text { alone. }\end{array}$ \\
\hline $\begin{array}{l}\text { Deshmukh, } 2017 \\
\text { [59] } \\
\text { India }\end{array}$ & Healthy & 15 & 15 & $\begin{array}{l}\text { Bifidobacterium, } \\
\text { Lactobacillus, } \\
\text { S. Boulardii }\end{array}$ & $\begin{array}{l}14 \text { days/ } \\
14 \text { days }\end{array}$ & Sachet & $\begin{array}{c}\text { Supragingival } \\
\text { scaling } \\
+ \\
\text { chlorhexidine } \\
\text { mouthwash control } \\
\end{array}$ & Yes & $\mathrm{PII} *, \mathrm{GI}^{*}$ & $\begin{array}{l}\text { Probiotic mouthwashes have } \\
\text { similar efficacy to chlorhexidine } \\
\text { and are a potential alternative } \\
\text { with fewer side effects. }\end{array}$ \\
\hline $\begin{array}{c}\text { Dhaliwal, } 2017 \\
\text { [60] } \\
\text { India }\end{array}$ & Periodontitis & 14 & 13 & $\begin{array}{c}\text { B. mesentericus, } \\
\text { C. butyricum, } \\
\text { L. sporogenes, S. faecalis }\end{array}$ & $\begin{array}{l}21 \text { days/ } \\
30 \text { days }\end{array}$ & Lozenge & SRP & Not mentioned & $\begin{array}{l}\mathrm{PI}{ }^{*}, \mathrm{GI}{ }^{*}, \mathrm{PPD}^{*}, \mathrm{RAL}{ }^{*}, A . \\
\text { actinomycetemomitans, } P \text {. } \\
\text { gingivalis }^{+}, \text {P. intermedia }\end{array}$ & $\begin{array}{l}\text { Probiotics may be used as an } \\
\text { adjunctive treatment for the } \\
\text { management of chronic } \\
\text { periodontitis. }\end{array}$ \\
\hline $\begin{array}{l}\text { Duarte, } 2019[61] \\
\text { United Arab } \\
\text { Emirates }\end{array}$ & Gingivitis & 5 & 5 & $\begin{array}{l}\text { S. oralis, S. rattus, } \\
\text { S. uberis }\end{array}$ & $\begin{array}{l}30 \text { days/ } \\
30 \text { days }\end{array}$ & Mouthwash & $\begin{array}{l}\text { 1) SRP } \\
\text { 2) SRP + } \\
\text { chlorhexidine } \\
\text { mouthwash }\end{array}$ & Yes & $\mathrm{GI}^{*}, \mathrm{OHI}^{+}, \mathrm{PI}^{*}$ & $\begin{array}{l}\text { Changes may be attributed to } \\
\text { type and duration of intervention. } \\
\text { Probiotics showed similar efficacy } \\
\text { to chlorhexidine and better } \\
\text { results compared to SRP alone. }\end{array}$ \\
\hline
\end{tabular}


Table 1. Cont.

\begin{tabular}{|c|c|c|c|c|c|c|c|c|c|c|}
\hline \multirow{3}{*}{$\begin{array}{l}\text { Author, Year, } \\
\text { Country }\end{array}$} & \multicolumn{3}{|c|}{ Study Sample Characteristics } & \multirow{3}{*}{$\begin{array}{l}\text { Probiotic } \\
\text { Formulation }\end{array}$} & \multirow{3}{*}{$\begin{array}{l}\text { Treatment } \\
\text { Duration// } \\
\text { Immediate } \\
\text { Follow-Up }\end{array}$} & \multirow{3}{*}{$\begin{array}{l}\text { Mode of } \\
\text { Probiotic } \\
\text { Delivery }\end{array}$} & \multirow{3}{*}{ Other Treatments } & \multirow{3}{*}{$\begin{array}{l}\text { Oral Hygiene } \\
\text { Instructions }\end{array}$} & \multirow{3}{*}{ Outcomes Investigated } & \multirow{3}{*}{ Key Findings } \\
\hline & \multirow{2}{*}{ Disease Status } & \multicolumn{2}{|c|}{ Sample Size } & & & & & & & \\
\hline & & Probiotic & Control & & & & & & & \\
\hline $\begin{array}{c}\text { Elsadek, } 2020 \text { [62] } \\
\text { Saudi Arabia }\end{array}$ & Periodontitis & 19 & 19 & L. reuteri & $\begin{array}{l}3 \text { weeks/ } \\
12 \text { weeks }\end{array}$ & Lozenge & $\begin{array}{l}\text { 1) RSD + } \\
\text { Photodynamic } \\
\text { therapy } \\
\text { 2) RSD alone }\end{array}$ & Yes & $\begin{array}{c}\mathrm{CAL}^{*}, \mathrm{BOP}^{*}, \mathrm{PPD}^{*}, \mathrm{PS}^{*}, \\
\text { P. gingivalis }{ }^{+}, \text {T. Forsythia }^{+}, \\
\text {T. denticola }\end{array}$ & $\begin{array}{l}\text { Photodynamic therapy showed } \\
\text { greater benefits for deeper } \\
\text { periodontal pockets. } \\
\text { Probiotics reduced bacterial } \\
\text { counts more than RSD alone. }\end{array}$ \\
\hline $\begin{array}{l}\text { Ercan, } 2020[63] \\
\text { Turkey }\end{array}$ & Gingivitis & 40 & 40 & $\begin{array}{l}\text { B. lactis, B. longum, } \\
\text { E. faecium, } \\
\text { L. acidophilus, } \\
\text { L. plantarum, } \\
\text { S. thermophilus, }\end{array}$ & $\begin{array}{l}1 \text { month/ } \\
1 \text { month }\end{array}$ & Chewing tablet & SRP & Yes & $\begin{array}{c}\mathrm{PII}^{+}, \mathrm{GI}^{*}, \mathrm{GCF}^{*}, \mathrm{IL}^{-} 6^{+}, \\
\mathrm{IL} 8^{+}, \mathrm{IL} 10^{+}\end{array}$ & $\begin{array}{l}\text { Adjunct synbiotics improved } \\
\text { clinical and immunological } \\
\text { outcomes in gingivitis patients, } \\
\text { irrespective of smoking status. }\end{array}$ \\
\hline $\begin{array}{c}\text { Grusovin, } 2019 \\
\text { [64] } \\
\text { Italy }\end{array}$ & Periodontitis & 10 & 10 & L. reuteri & $\begin{array}{l}\text { 3 months, } \\
\text { 3-month } \\
\text { washout, } \\
3 \text { months/ } \\
3 \text { months, } \\
9 \text { months }\end{array}$ & Lozenge & FM-GBT & Yes & $\begin{array}{c}\mathrm{BOP}^{+}, \mathrm{PPD}^{+}, \mathrm{PAL}^{+}, \text {tooth } \\
\text { survival }\end{array}$ & $\begin{array}{l}\text { Probiotics improved clinical } \\
\text { parameters with periodontal } \\
\text { maintenance therapy. }\end{array}$ \\
\hline $\begin{array}{c}\text { Hallström, } 2013 \\
\text { [42] } \\
\text { Sweden }\end{array}$ & Healthy & 9 & 9 & L. reuteri & $\begin{array}{l}3 \text { weeks/ } \\
3 \text { weeks }\end{array}$ & Lozenge & None & No & $\begin{array}{l}\text { PI, GI, BOP, GCF, IL-1 } \beta * \text { *, } \\
\text { IL6, IL8*, IL10, IL-18*, } \\
\text { MII-1 } \beta \text { *, TNF- } \alpha, \\
\text { A. actinomycetemcomitan, } \\
\text { A. naeslundii, C. rectus, } \\
\text { F. alocis, F. nucleatum *, } \\
\text { L. reuteri, L. fermentum, } \\
\text { P. micra, P. endodontis, } \\
\text { P. intermedia, P. gingivalis, } \\
\text { S. intermedia, S. mutans, } \\
\text { S. oralis *, S. sanguinis, } \\
\text { T. forsythia, T. denticola, } \\
\text { V. parvula * }\end{array}$ & $\begin{array}{l}\text { Probiotic supplementation did } \\
\text { not significantly affect plaque } \\
\text { accumulation, inflammatory } \\
\text { reactions in the gingiva, and the } \\
\text { microbiological composition in } \\
\text { healthy individuals with } \\
\text { experimental gingivitis. }\end{array}$ \\
\hline $\begin{array}{l}\text { Ikram, } 2018 \text { [65] } \\
\quad \text { Pakistan }\end{array}$ & Periodontitis & 15 & 15 & L. reuteri & $\begin{array}{l}3 \text { months/ } \\
4 \text { months }\end{array}$ & Sachet & $\begin{array}{l}\mathrm{SRP}+\text { amoxicillin }+ \\
\text { metronidazole }\end{array}$ & Yes & $\mathrm{PII}^{*}, \mathrm{CAL}^{*}, \mathrm{BOP} *, \mathrm{PPD}^{*}$ & $\begin{array}{l}\text { Probiotics showed similar efficacy } \\
\text { in the improvement of } \\
\text { periodontal clinical outcomes as } \\
\text { antibiotics. }\end{array}$ \\
\hline $\begin{array}{l}\text { Ikram, } 2019[66] \\
\quad \text { Pakistan }\end{array}$ & Periodontitis & 14 & 14 & L. reuteri & $\begin{array}{l}12 \text { weeks/ } \\
12 \text { weeks }\end{array}$ & Sachet & SRP & Yes & $\mathrm{PII}^{*}, \mathrm{CAL}^{+}, \mathrm{BOP}^{+}, \mathrm{PPD}^{+}$ & $\begin{array}{l}\text { Probiotics may be used as an } \\
\text { adjunctive treatment with SRP to } \\
\text { treat chronic periodontitis. }\end{array}$ \\
\hline $\begin{array}{l}\text { Ince, } 2015 \text { [67] } \\
\text { Turkey }\end{array}$ & Periodontitis & 15 & 15 & L. reuteri & $\begin{array}{l}3 \text { weeks/ } \\
3 \text { weeks }\end{array}$ & Lozenge & SRP & Yes & $\begin{array}{c}\mathrm{PII}^{+}, \mathrm{GI}^{+}, \mathrm{BOP}^{+}, \mathrm{PPD}^{+}, \\
\mathrm{CAL}^{+}, \mathrm{GCF}^{*}, \mathrm{MMP}^{+}{ }^{+}, \\
\mathrm{TIMP}^{+}{ }^{+}\end{array}$ & $\begin{array}{l}\text { Adjuvant probiotic treatment } \\
\text { improved clinical and } \\
\text { immunological outcomes in } \\
\text { periodontitis patients. }\end{array}$ \\
\hline
\end{tabular}


Table 1. Cont.

\begin{tabular}{|c|c|c|c|c|c|c|c|c|c|c|}
\hline \multirow{3}{*}{$\begin{array}{l}\text { Author, Year, } \\
\text { Country }\end{array}$} & \multicolumn{3}{|c|}{ Study Sample Characteristics } & \multirow{3}{*}{$\begin{array}{l}\text { Probiotic } \\
\text { Formulation }\end{array}$} & \multirow{3}{*}{$\begin{array}{l}\text { Treatment } \\
\text { Duration/ } \\
\text { Immediate } \\
\text { Follow-Up }\end{array}$} & \multirow{3}{*}{$\begin{array}{l}\text { Mode of } \\
\text { Probiotic } \\
\text { Delivery }\end{array}$} & \multirow{3}{*}{ Other Treatments } & \multirow{3}{*}{$\begin{array}{l}\text { Oral Hygiene } \\
\text { Instructions }\end{array}$} & \multirow{3}{*}{ Outcomes Investigated } & \multirow{3}{*}{ Key Findings } \\
\hline & \multirow{2}{*}{ Disease Status } & \multicolumn{2}{|c|}{ Sample Size } & & & & & & & \\
\hline & & Probiotic & Control & & & & & & & \\
\hline $\begin{array}{l}\text { Iniesta, } 2012[43] \\
\text { Spain }\end{array}$ & Gingivitis & 20 & 20 & L. reuteri & $\begin{array}{l}4 \text { weeks/ } \\
4 \text { weeks }\end{array}$ & Chewing tablet & None & No & $\begin{array}{l}\text { PII, GI, Lactobacillus spp., } \\
\text { A. actinomycetemcomitan, } \\
\text { C. rectus, } \\
\text { Capnocytophaga spp., } \\
\text { E. corrodens, F. nucleatum, } \\
\text { P. micra, P. intermedia, } \\
\text { P. gingivalis, } \\
\text { Tannerella forsythia, total } \\
\text { bacteria }\end{array}$ & $\begin{array}{l}\text { Probiotic administration reduced } \\
\text { subgingival periodontopathogen } \\
\text { count. }\end{array}$ \\
\hline $\begin{array}{l}\text { Invernici, } 2018 \\
{[68]} \\
\text { Brazil }\end{array}$ & Periodontitis & 20 & 21 & B. lactis & $\begin{array}{l}30 \text { days/ } \\
30 \text { days }\end{array}$ & Lozenge & SRP & Yes & $\begin{array}{l}\mathrm{PII}^{+}, \mathrm{CAL}^{+}, \mathrm{PPD}^{+}, \mathrm{BOP} \\
{ }^{+}, \mathrm{REC}, \mathrm{IL}-1 \beta^{+}, \mathrm{IL}_{-}^{+} 8^{+}, \\
\quad \mathrm{IL}^{*} 0^{*}, \text { B. animalis }^{+},\end{array}$ & $\begin{array}{l}\text { Probiotic supplementation in } \\
\text { addition to SRP may improve } \\
\text { clinical, microbiological, and } \\
\text { immunological outcomes in } \\
\text { generalized chronic periodontitis } \\
\text { patients. }\end{array}$ \\
\hline $\begin{array}{l}\text { Iwasakia, } 2016 \text { [69] } \\
\text { Japan }\end{array}$ & Periodontitis & 19 & 17 & L. plantarum & $\begin{array}{l}12 \text { weeks/ } \\
12 \text { weeks }\end{array}$ & Capsule & SPT & Not mentioned & PII, GI, BOP, PPD ${ }^{+}$ & $\begin{array}{l}\text { Chronic periodontitis patients } \\
\text { with adjunctive probiotic } \\
\text { treatment may lead to } \\
\text { improvements in periodontal } \\
\text { pockets. }\end{array}$ \\
\hline $\begin{array}{l}\text { Jäsberg, } 2018[71] \\
\text { Finland }\end{array}$ & Healthy & 29 & 31 & $\begin{array}{l}\text { B. animalis, } \\
\text { L. rhamnosus }\end{array}$ & $\begin{array}{l}4 \text { weeks/ } \\
4 \text { weeks }\end{array}$ & Lozenge & None & Not mentioned & $\begin{array}{l}\mathrm{PII}^{+}, \mathrm{GI}^{+}, \mathrm{MMP}^{+} 8, \\
\mathrm{MMP}^{+}{ }^{+}, \mathrm{TIMP}^{+} 1^{+} \\
\text {S. mutans, lactobacilli }\end{array}$ & $\begin{array}{l}\text { Probiotics may immunomodulate } \\
\text { the oral cavity. }\end{array}$ \\
\hline $\begin{array}{l}\text { Keller, } 2018 \text { [72] } \\
\text { Denmark }\end{array}$ & Gingivitis & 23 & 24 & $\begin{array}{l}\text { L. curvatus, } \\
\text { L. rhamnosus }\end{array}$ & $\begin{array}{l}4 \text { weeks/ } \\
4 \text { weeks }\end{array}$ & Tablet & None & No & $\begin{array}{l}\mathrm{PII}^{*}, \mathrm{BOP}^{+}, \mathrm{GCF}^{+}, \\
\text {IL-1 } \beta, \text { IL-6, IL-8, IL-10, } \\
\text { TNF- } \alpha\end{array}$ & $\begin{array}{l}\text { Probiotic use may improve } \\
\text { gingival health without affecting } \\
\text { the oral microbiome and immune } \\
\text { response. }\end{array}$ \\
\hline $\begin{array}{l}\text { Krasse, } 2005 \text { [73] } \\
\text { Sweden }\end{array}$ & Gingivitis & 20 & 18 & L. reuteri & $\begin{array}{l}14 \text { days/ } \\
14 \text { days }\end{array}$ & Chewing gum & None & Yes & $\begin{array}{l}\text { PII }^{+}, \mathrm{GI}^{+}, \text {L. reuteri }^{+}, \\
\text {Total lactobacillus } \\
\end{array}$ & $\begin{array}{l}\text { L. reuteri can reduce PII and GI in } \\
\text { gingivitis patients. }\end{array}$ \\
\hline $\begin{array}{l}\text { Kuka, } 2019[74] \\
\text { Turkey }\end{array}$ & Periodontitis & 18 & 18 & L. reuteri & $\begin{array}{l}3 \text { weeks/ } \\
12 \text { weeks }\end{array}$ & Tablet & IPT & Yes & $\begin{array}{c}\mathrm{BOP}^{+}, \mathrm{PPD}^{+}, \mathrm{GCF}^{+}, \\
\mathrm{NO}^{+}\end{array}$ & $\begin{array}{l}\text { Probiotics may be an adjunct to } \\
\text { IPT. NO in GCF is a potential } \\
\text { inflammatory marker in } \\
\text { periodontal diseases. }\end{array}$ \\
\hline
\end{tabular}


Table 1. Cont.

\begin{tabular}{|c|c|c|c|c|c|c|c|c|c|c|}
\hline \multirow{3}{*}{$\begin{array}{l}\text { Author, Year, } \\
\text { Country }\end{array}$} & \multicolumn{3}{|c|}{ Study Sample Characteristics } & \multirow{3}{*}{$\begin{array}{l}\text { Probiotic } \\
\text { Formulation }\end{array}$} & \multirow{3}{*}{$\begin{array}{l}\text { Treatment } \\
\text { Duration/ } \\
\text { Immediate } \\
\text { Follow-Up }\end{array}$} & \multirow{3}{*}{$\begin{array}{l}\text { Mode of } \\
\text { Probiotic } \\
\text { Delivery }\end{array}$} & \multirow{3}{*}{ Other Treatments } & \multirow{3}{*}{$\begin{array}{l}\text { Oral Hygiene } \\
\text { Instructions }\end{array}$} & \multirow{3}{*}{ Outcomes Investigated } & \multirow{3}{*}{ Key Findings } \\
\hline & \multirow{2}{*}{ Disease Status } & \multicolumn{2}{|c|}{ Sample Size } & & & & & & & \\
\hline & & Probiotic & Control & & & & & & & \\
\hline $\begin{array}{l}\text { Kuru, } 2017 \text { [75] } \\
\text { Turkey }\end{array}$ & Healthy & 26 & 25 & B. animalis & $\begin{array}{l}4 \text { weeks/ } \\
4 \text { weeks }\end{array}$ & Yogurt & None & Yes & $\begin{array}{c}\mathrm{PII}^{+}, \mathrm{GI}^{+}, \mathrm{BOP}^{+}, \mathrm{PPD}^{+}, \\
\quad \mathrm{GCF}^{+}, \mathrm{IL}-1 \beta^{+}\end{array}$ & $\begin{array}{l}\text { Probiotics improved clinical and } \\
\text { immunological outcomes } \\
\text { compared to controls after a } \\
\text { 5-day non-brushing period. }\end{array}$ \\
\hline $\begin{array}{l}\text { Laleman, } 2015 \text { [76] } \\
\text { Turkey }\end{array}$ & Periodontitis & 24 & 24 & $\begin{array}{l}\text { S. oralis, S. rattus, } \\
\text { S. uberis }\end{array}$ & $\begin{array}{l}12 \text { weeks/ } \\
12 \text { weeks }\end{array}$ & Tablet & SRP & Not mentioned & $\begin{array}{l}\mathrm{CAL}^{*}, \mathrm{BOP} *, \mathrm{PPD}^{*}, \\
\text { REC *, F. nucleatum *, } \\
\text { P. gingivalis *, } \\
\text { P. intermedia }{ }^{+}, \\
\text {T. forsythia * }\end{array}$ & $\begin{array}{l}\text { Probiotic formulation used did } \\
\text { not show statistically significant } \\
\text { improvements in clinical or } \\
\text { microbiological outcomes. }\end{array}$ \\
\hline $\begin{array}{l}\text { Laleman, } 2019 \text { [77] } \\
\text { Belgium }\end{array}$ & Periodontitis & 19 & 20 & L. reuteri & $\begin{array}{l}12 \text { weeks/ } \\
12 \text { weeks }\end{array}$ & Lozenge & NSPT & Yes & $\begin{array}{c}\mathrm{PII}^{*}, \mathrm{CAL}^{*}, \mathrm{BOP} * \\
\text { PPD }^{+}, \mathrm{REC}^{*}, \\
\text { A. actinomycetemcomitans, } \\
\text { F. nucleatum, P. intermedia, } \\
\text { P. gingivalis }\end{array}$ & $\begin{array}{l}\text { Adjunctive use of probiotics after } \\
\text { NSPT reduced PPD and the } \\
\text { percentage of sites in need of } \\
\text { surgery. }\end{array}$ \\
\hline $\begin{array}{l}\text { Lee, } 2015[78] \\
\quad \text { Korea }\end{array}$ & Healthy & 14 & 16 & L. brevis & $\begin{array}{l}14 \text { days/ } \\
14 \text { days }\end{array}$ & Lozenge & $\begin{array}{l}\text { Scaling and } \\
\text { polishing }\end{array}$ & Yes & $\begin{array}{l}\text { PII *, GI *, BOP *, NO, } \\
\text { MMP-8, PGE2 * }\end{array}$ & $\begin{array}{c}\text { Probiotic supplementation may } \\
\text { decrease inflammatory cascades } \\
\text { through NO and PGE2. }\end{array}$ \\
\hline $\begin{array}{c}\text { Mayanagi, } 2009 \\
{[79]} \\
\text { Japan }\end{array}$ & Periodontitis & 34 & 32 & L. salivarius & $\begin{array}{l}8 \text { weeks/ } \\
8 \text { weeks }\end{array}$ & Tablet & None & No & $\begin{array}{l}\text { A. actinomycetemcomitans, } \\
\text { P. intermedia, } P \text {. gingivalis, } \\
\text { T. forsythia }{ }^{+}, \text {T. denticola, } \\
\text { total bacteria * }\end{array}$ & $\begin{array}{c}\text { Probiotics decreased the } \\
\text { subgingival T. forsythia count at } 4 \\
\text { and } 8 \text { weeks and the total bacteria } \\
\text { count at } 4 \text { weeks. }\end{array}$ \\
\hline $\begin{array}{c}\text { Meenakshi, } 2018 \\
\text { [80] } \\
\text { India }\end{array}$ & Periodontitis & 10 & 10 & L. casei & $\begin{array}{l}1 \text { month/ } \\
1 \text { month }\end{array}$ & Drink & SRP & No & $\begin{array}{l}\mathrm{PII}^{+}, \mathrm{GI}^{+}, \mathrm{CAL}^{+}, \mathrm{PPD}^{+}, \\
\text {total } \text { bacteria }^{+}\end{array}$ & $\begin{array}{l}\text { Probiotics as an adjunct to SRP } \\
\text { improved clinical outcomes and } \\
\text { reduced total bacterial count. }\end{array}$ \\
\hline $\begin{array}{l}\text { Mitic, } 2017 \text { [81] } \\
\text { Macedonia }\end{array}$ & Periodontitis & 15 & 15 & $\begin{array}{l}\text { B. bifidum, } \\
\text { B. coagulans, } \\
\text { L. acidophilus, } \\
\text { L. bulgaricus, } \\
\text { S. thermophilus }\end{array}$ & $\begin{array}{l}15 \text { days / } \\
1 \text { month }\end{array}$ & Lozenge & SRP & Yes & $\begin{array}{l}\text { PII *, GI *, GBI *, CAL *, } \\
\text { PPD }^{+} \text {, anaerobic } \\
\text { bacterial count }^{+}\end{array}$ & $\begin{array}{l}\text { Probiotics may improve clinical } \\
\text { outcomes and bacterial count in } \\
\text { periodontitis patients. }\end{array}$ \\
\hline $\begin{array}{l}\text { Montero, } 2017 \text { [14] } \\
\text { Spain }\end{array}$ & Gingivitis & 30 & 29 & $\begin{array}{l}\text { L. brevis, L. plantarum, } \\
\text { P. acidilactici }\end{array}$ & $\begin{array}{l}6 \text { weeks/ } \\
6 \text { weeks }\end{array}$ & Chewing tablet & PMPR & Yes & $\begin{array}{l}\text { PII *, GI *, AngBs }{ }^{+}, \\
\text {A. actinomycetemcomitans *, } \\
\text { C. rectus, Fusobacterium } \\
\text { spp., P. gingivalis, } \\
\text { T. forsythia * }\end{array}$ & $\begin{array}{l}\text { Decreased number of sites with } \\
\text { severe inflammation compared to } \\
\text { placebo group in gingivitis } \\
\text { patients. Decreased T. forsythia } \\
\text { count. }\end{array}$ \\
\hline $\begin{array}{l}\text { Morales, } 2016 \text { [82] } \\
\text { Chile }\end{array}$ & Periodontitis & 14 & 14 & L. rhamnosus & $\begin{array}{l}3 \text { months/ } \\
3 \text { months }\end{array}$ & Sachet & SRP & Yes & $\mathrm{CAL}^{*}, \mathrm{PII} *$, BOP, $\mathrm{PPD} *$ & $\begin{array}{l}\text { Probiotic use improved clinical } \\
\text { symptoms similar to SRP alone. }\end{array}$ \\
\hline
\end{tabular}


Table 1. Cont.

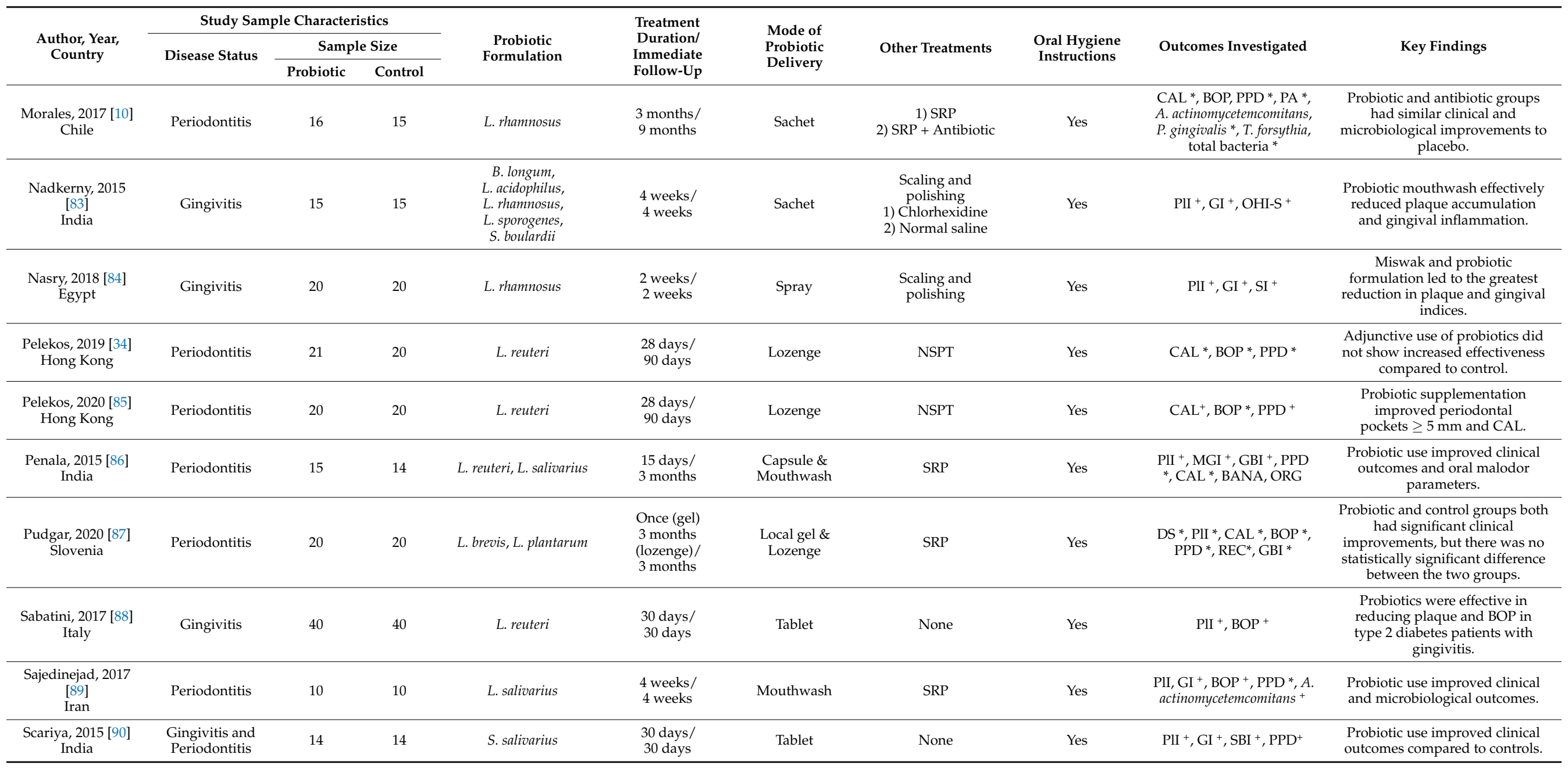


Table 1. Cont.

\begin{tabular}{|c|c|c|c|c|c|c|c|c|c|c|}
\hline \multirow{3}{*}{$\begin{array}{l}\text { Author, Year, } \\
\text { Country }\end{array}$} & \multicolumn{3}{|c|}{ Study Sample Characteristics } & \multirow{3}{*}{$\begin{array}{l}\text { Probiotic } \\
\text { Formulation }\end{array}$} & \multirow{3}{*}{$\begin{array}{l}\text { Treatment } \\
\text { Duration/ } \\
\text { Immediate } \\
\text { Follow-Up }\end{array}$} & \multirow{3}{*}{$\begin{array}{l}\text { Mode of } \\
\text { Probiotic } \\
\text { Delivery }\end{array}$} & \multirow{3}{*}{ Other Treatments } & \multirow{3}{*}{$\begin{array}{l}\text { Oral Hygiene } \\
\text { Instructions }\end{array}$} & \multirow{3}{*}{ Outcomes Investigated } & \multirow{3}{*}{ Key Findings } \\
\hline & \multirow{2}{*}{ Disease Status } & \multicolumn{2}{|c|}{ Sample Size } & & & & & & & \\
\hline & & Probiotic & Control & & & & & & & \\
\hline $\begin{array}{l}\text { Schlagenhauf, } \\
\text { 2018 [91] } \\
\text { Germany }\end{array}$ & Gingivitis & 24 & 21 & L. reuteri & $\begin{array}{l}\text { Within } 2 \text { days } \\
\text { after delivery } \\
(41.9 \pm 16.0 \text { days })\end{array}$ & Lozenge & None & No & $\mathrm{PII}^{+}, \mathrm{GI}^{+}, \mathrm{TNF}-\alpha$ & $\begin{array}{l}\text { Probiotics may be a useful } \\
\text { adjunct for pregnancy-related } \\
\text { gingivitis. }\end{array}$ \\
\hline $\begin{array}{l}\text { Schlagenhauf, } \\
2020[92] \\
\text { Germany }\end{array}$ & $\begin{array}{l}\text { Gingivitis \& } \\
\text { Periodontitis }\end{array}$ & 33 & 35 & L. reuteri & $\begin{array}{l}42 \text { days/ } \\
42 \text { days }\end{array}$ & Lozenge & None & No & $\underset{+, \mathrm{PCR}^{+}, \mathrm{GI}^{+}, \mathrm{BOP}^{+}+, \mathrm{PAL}}{\mathrm{PPD}^{+}}$ & $\begin{array}{l}\text { Probiotic use improved all clinical } \\
\text { outcomes compared to controls. }\end{array}$ \\
\hline $\begin{array}{l}\text { Shah, } 2013 \text { [93] } \\
\text { India }\end{array}$ & Periodontitis & 10 & $\begin{array}{c}10 \\
\text { (Control) } \\
10 \text { (An- } \\
\text { tibiotic) }\end{array}$ & L. brevis & $\begin{array}{l}2 \text { weeks/ } \\
2 \text { months }\end{array}$ & Tablet & $\begin{array}{l}\text { SRP } \\
\text { 1) Probiotic }+ \\
\text { Doxycycline } \\
\text { 2) Doxycycline alone }\end{array}$ & No & $\begin{array}{c}\mathrm{PII}{ }^{*}, \mathrm{GI}^{*}, \mathrm{CAL}^{*}, \mathrm{PPD}^{*}, \\
\text { lactobacilli }{ }^{+}, \\
\text {A. actinomycetemcomitans }\end{array}$ & $\begin{array}{l}\text { Probiotic use decreased clinical } \\
\text { and microbiological parameters } \\
\text { when used alone or in } \\
\text { combination with doxycycline. }\end{array}$ \\
\hline $\begin{array}{l}\text { Shah, } 2017 \text { [94] } \\
\text { India }\end{array}$ & Periodontitis & 6 & 6 & L. brevis & $\begin{array}{l}14 \text { days/ } \\
5 \text { months }\end{array}$ & Lozenge & $\begin{array}{l}\text { SRP } \\
\text { 1) Probiotics + } \\
\text { Doxycycline } \\
\text { 2) Doxycycline alone }\end{array}$ & No & $\begin{array}{l}\mathrm{GI}^{+}, \mathrm{PII}, \mathrm{PPD}, \mathrm{CAL}, \\
\text { A. actinomycetemcomitans, } \\
\text { Lactobacillus spp. }\end{array}$ & $\begin{array}{l}\text { No synergy at } 5 \text { months when } \\
\text { probiotics and doxycycline were } \\
\text { both given. } \\
\text { No statistically significant } \\
\text { difference between antibiotic and } \\
\text { probiotic supplementation. }\end{array}$ \\
\hline $\begin{array}{l}\text { Shetty, } 2020[95] \\
\text { India }\end{array}$ & Periodontitis & 60 & 60 & $\begin{array}{l}\text { B. mesentericus, } \\
\text { C. butyricum, } \\
\text { L. sporogenes. } \\
\text { S. faecalis }\end{array}$ & $\begin{array}{l}\text { Once (local)/ } \\
3 \text { months }\end{array}$ & Local & SRP & Not mentioned & $\begin{array}{c}\mathrm{PII} *, \mathrm{GI}^{*}, \mathrm{PPD}^{*}, \mathrm{IL}^{-6+}{ }^{+}, \\
\text {ALP*, } \\
\text { P.Gingivalis } \\
\text { P. intermedia }\end{array}$ & $\begin{array}{l}\text { Synbiotic treatment may improve } \\
\text { clinical, microbiological, and } \\
\text { immunological outcomes in } \\
\text { patients with chronic } \\
\text { periodontitis. }\end{array}$ \\
\hline $\begin{array}{c}\text { Shimauchi, } 2008 \\
{[96]} \\
\text { Japan }\end{array}$ & Healthy & 34 & 32 & L. salivarius & $\begin{array}{l}8 \text { weeks/ } \\
8 \text { weeks }\end{array}$ & Tablet & None & No & $\begin{array}{l}\text { PII *, GI *, BOP *, PPD *, } \\
\text { L. salivarius }{ }^{+}, \\
\text {Lactoferritin * (Saliva) }\end{array}$ & $\begin{array}{l}\text { Probiotics may be useful for } \\
\text { maintenance and/or } \\
\text { improvement of oral health in } \\
\text { individuals at risk of periodontal } \\
\text { diseases. }\end{array}$ \\
\hline $\begin{array}{c}\text { Sinkiewicz, } 2010 \\
{[97]} \\
\text { Sweden }\end{array}$ & Healthy & 11 & 12 & L. reuteri & $\begin{array}{l}12 \text { weeks/ } \\
12 \text { weeks }\end{array}$ & Chewing gum & None & No & $\begin{array}{l}\text { PII, A. naeslundii*, } \\
\text { A. actinomycetemcomitans *, } \\
\text { C. rectus }{ }^{*}, \text { F. alocis *, } \\
\text { F. nucleatum **, } \\
\text { L. acidophilus, } \\
\text { L. fermentum }{ }^{*}, \text { L. reuteri *, } \\
\text { P. micra, P. gingivalis *, } \\
\text { P. endodontalis *, } \\
\text { P. intermedia *, } \\
\text { T. forsythia *, T. denticola *, } \\
\text { S. intermedia, S. mutans *, } \\
\text { S. oralis, S. sanguinis *, } \\
\text { V. parvula * }\end{array}$ & $\begin{array}{l}\text { There was a statistically } \\
\text { significant increase in plaque in } \\
\text { the controls, but not the } \\
\text { probiotics group. No changes } \\
\text { between probiotics and control } \\
\text { groups in the oral microbiota. }\end{array}$ \\
\hline $\begin{array}{l}\text { Slawik, } 2011[98] \\
\text { Germany }\end{array}$ & Healthy & 11 & 17 & L. casei & $\begin{array}{l}14 \text { days/ } \\
14 \text { days }\end{array}$ & Drink & None & No & $\mathrm{PII} *, \mathrm{GI}^{*}, \mathrm{BOP}^{+}, \mathrm{GCF}^{+}$ & $\begin{array}{l}\text { Probiotics may have an } \\
\text { anti-inflammatory effect. }\end{array}$ \\
\hline
\end{tabular}


Table 1. Cont.

\begin{tabular}{|c|c|c|c|c|c|c|c|c|c|c|}
\hline \multirow{3}{*}{$\begin{array}{l}\text { Author, Year, } \\
\text { Country }\end{array}$} & \multicolumn{3}{|c|}{ Study Sample Characteristics } & \multirow{3}{*}{$\begin{array}{l}\text { Probiotic } \\
\text { Formulation }\end{array}$} & \multirow{3}{*}{$\begin{array}{l}\text { Treatment } \\
\text { Duration/ } \\
\text { Immediate } \\
\text { Follow-Up }\end{array}$} & \multirow{3}{*}{$\begin{array}{l}\text { Mode of } \\
\text { Probiotic } \\
\text { Delivery }\end{array}$} & \multirow{3}{*}{ Other Treatments } & \multirow{3}{*}{$\begin{array}{l}\text { Oral Hygiene } \\
\text { Instructions }\end{array}$} & \multirow{3}{*}{ Outcomes Investigated } & \multirow{3}{*}{ Key Findings } \\
\hline & \multirow{2}{*}{ Disease Status } & \multicolumn{2}{|c|}{ Sample Size } & & & & & & & \\
\hline & & Probiotic & Control & & & & & & & \\
\hline $\begin{array}{c}\text { Snulingga, } 2020 \\
\text { [99] } \\
\text { Indonesia } \\
\end{array}$ & Periodontitis & 8 & 8 & L. reuteri & $\begin{array}{l}14 \text { days/ } \\
14 \text { days }\end{array}$ & Lozenge & SRP & Not mentioned & $\mathrm{CAL}^{+}, \mathrm{IL}_{-4}$ & $\begin{array}{l}\text { Probiotic use as an adjunct } \\
\text { decreased CAL and increased } \\
\text { IL-4. }\end{array}$ \\
\hline $\begin{array}{l}\text { Staab, } 2009[100] \\
\text { Germany }\end{array}$ & Healthy & 25 & 25 & L. casei & $\begin{array}{l}8 \text { weeks/ } \\
8 \text { weeks }\end{array}$ & Drink & None & No & $\begin{array}{l}\mathrm{PII}^{+}, \mathrm{PBI}^{*}, \mathrm{MPO}^{+}, \\
\mathrm{MMP}^{-}{ }^{+}, \text {Elastase }\end{array}$ & $\begin{array}{l}\text { Probiotics may improve } \\
\text { periodontal health through } \\
\text { immunomodulation. }\end{array}$ \\
\hline $\begin{array}{l}\text { Suzuki, } 2012[101] \\
\text { Japan }\end{array}$ & Periodontitis & 20 & 22 & L. salivarius & $\begin{array}{l}2 \text { weeks/ } \\
2 \text { weeks }\end{array}$ & Oil drops & None & No & $\begin{array}{c}\text { BOP }{ }^{+}, \mathrm{PPD}^{*}, \\
\text { Ubiquitous bacteria }{ }^{*}, \\
\text { F. nucleatum, } P \text {. gingivalis, } \\
\text { L. salivarius }{ }^{*}, \\
\text { P. intermedia, S. mutans, } \\
\text { T. forsythia, T. denticola }\end{array}$ & $\begin{array}{l}\text { Probiotics improved BOP and } \\
\text { had a decreased } \\
\text { periodontopathogen count } \\
\text { compared to controls. }\end{array}$ \\
\hline $\begin{array}{l}\text { Tekce, } 2015[41] \\
\text { Turkey }\end{array}$ & Periodontitis & 20 & 20 & L. reuteri & $\begin{array}{l}3 \text { weeks/ } \\
3 \text { weeks }\end{array}$ & Lozenge & SRP & Yes & $\begin{array}{l}\mathrm{PII}^{+}, \mathrm{GI}^{+}, \mathrm{BOP}^{+}, \mathrm{PPD}^{+}, \\
\mathrm{RAL}^{*}, \text { Anaerobic } \\
\text { bacteria }^{+}, \mathrm{TVC}^{+}\end{array}$ & $\begin{array}{l}\text { Probiotics as an adjuvant can } \\
\text { improve clinical and } \\
\text { microbiological outcomes. }\end{array}$ \\
\hline $\begin{array}{l}\text { Teughels, } 2013 \\
{[102]} \\
\text { Turkey }\end{array}$ & Periodontitis & 15 & 15 & L. reuteri & $\begin{array}{l}12 \text { weeks/ } \\
12 \text { weeks }\end{array}$ & Lozenge & SRP & Yes & $\begin{array}{l}\mathrm{PII}^{*}, \mathrm{CAL}^{+}, \mathrm{GBI}^{+}, \mathrm{BOP} * \\
\mathrm{PPD}^{*}, \mathrm{REC}^{*}, \\
\text { A. actinomycetemcomitans *, } \\
\text { F. nucleatum }{ }^{*}, \\
\text { T. forsythia }{ }^{*}, \\
\text { P. gingivalis }{ }^{+}, \\
\text {P. intermedia }{ }^{*}, \\
\text { Total bacteria }{ }^{*}\end{array}$ & $\begin{array}{l}\text { Probiotics as an adjuvant can } \\
\text { improve clinical and } \\
\text { microbiological outcomes. }\end{array}$ \\
\hline $\begin{array}{c}\text { Theodoro, } 2019 \\
\text { [103] } \\
\text { Brazil }\end{array}$ & Periodontitis & 14 & 14 & L. reuteri & $\begin{array}{l}21 \text { days/ } \\
90 \text { days }\end{array}$ & Chewing tablet & SRP & Yes & $\mathrm{BOP}^{+}, \mathrm{CAL} \mathrm{PPD}^{+}, \mathrm{REC}$ & $\begin{array}{l}\text { Adjuvant use of probiotics to trea } \\
\text { chronic periodontitis in smokers } \\
\text { reduced gingival inflammation. }\end{array}$ \\
\hline $\begin{array}{l}\text { Tobita, } 2018[104] \\
\text { Japan }\end{array}$ & Healthy & 8 & 8 & L. crispatus & $\begin{array}{l}4 \text { weeks/ } \\
4 \text { weeks }\end{array}$ & Tablet & None & No & $\begin{array}{c}\text { PS }^{+}, \\
\text {A. actinomycetemcomitans, } \\
\text { F. nucleatum }{ }^{*}, \text { T. forsythia, } \\
\text { P. gingivalis } \\
\text { P. intermedia, T. denticola. }\end{array}$ & $\begin{array}{l}\text { Probiotic use can improve the } \\
\text { oral environment and hence may } \\
\text { help prevent periodontal disease }\end{array}$ \\
\hline $\begin{array}{c}\text { Toiviainen, } 2015 \\
\text { [105] } \\
\text { Finland }\end{array}$ & Healthy & 29 & 31 & B. lactis, L. rhamnosus & $\begin{array}{l}4 \text { weeks/ } \\
4 \text { weeks }\end{array}$ & Lozenge & None & Not mentioned & $\begin{array}{l}\mathrm{PII}^{*}, \mathrm{GI}^{*}, \text { Lactobacillus, } \\
\text { S. mutans }\end{array}$ & $\begin{array}{l}\text { Probiotics improved clinical } \\
\text { outcomes but not microbiological }\end{array}$ \\
\hline $\begin{array}{c}\text { Twetman, } 2009 \\
\text { [106] } \\
\text { Denmark }\end{array}$ & Gingivitis & 14 & 13 & L. reuteri & $\begin{array}{l}2 \text { weeks/ } \\
2 \text { weeks }\end{array}$ & Chewing gum & None & Yes & $\begin{array}{l}\text { BOP*, IL-1 } \beta, \text { TNF- } \alpha, \\
\text { GCF }^{*} \text {, IL-6 } 6^{*} \text {, IL-8* , IL-10 }\end{array}$ & $\begin{array}{l}\text { Probiotics are beneficial to } \\
\text { gingival health in a dose } \\
\text { dependent manner. }\end{array}$ \\
\hline $\begin{array}{l}\text { Vicario, } 2013 \text { [107] } \\
\text { Spain }\end{array}$ & Periodontitis & 10 & 9 & L. reuteri & $\begin{array}{l}1 \text { month/ } \\
1 \text { month }\end{array}$ & Tablet & None & Yes & $\mathrm{PII}^{*}, \mathrm{BOP}^{*}, \mathrm{PPD}^{*}$ & $\begin{array}{l}\text { Probiotic supplementation can } \\
\text { improve inflammatory and } \\
\text { clinical outcomes in patients with } \\
\text { mild to moderate periodontitis. }\end{array}$ \\
\hline
\end{tabular}


Table 1. Cont.

\begin{tabular}{|c|c|c|c|c|c|c|c|c|c|c|}
\hline \multirow{3}{*}{$\begin{array}{l}\text { Author, Year, } \\
\text { Country }\end{array}$} & \multicolumn{3}{|c|}{ Study Sample Characteristics } & \multirow{3}{*}{$\begin{array}{l}\text { Probiotic } \\
\text { Formulation }\end{array}$} & \multirow{3}{*}{$\begin{array}{l}\text { Treatment } \\
\text { Duration/ } \\
\text { Immediate } \\
\text { Follow-Up }\end{array}$} & \multirow{3}{*}{$\begin{array}{l}\text { Mode of } \\
\text { Probiotic } \\
\text { Delivery }\end{array}$} & \multirow{3}{*}{ Other Treatments } & \multirow{3}{*}{$\begin{array}{l}\text { Oral Hygiene } \\
\text { Instructions }\end{array}$} & \multirow{3}{*}{ Outcomes Investigated } & \multirow{3}{*}{ Key Findings } \\
\hline & \multirow{2}{*}{ Disease Status } & \multicolumn{2}{|c|}{ Sample Size } & & & & & & & \\
\hline & & Probiotic & Control & & & & & & & \\
\hline $\begin{array}{l}\text { Vivekananda, } \\
2010[108] \\
\text { India }\end{array}$ & Periodontitis & 15 & 15 & L. reuteri & $\begin{array}{l}21 \text { days/ } \\
42 \text { days }\end{array}$ & Lozenge & $\begin{array}{l}\text { 1) SRP } \\
\text { 2) Without SRP }\end{array}$ & Yes & $\begin{array}{l}\mathrm{PII}^{*}, \mathrm{GI}^{*}, \mathrm{GBI}^{*}, \mathrm{CAL}^{*}, \\
\text { PPD }^{*}, A . \\
\text { actinomycetemcomitans } \\
\text { P. gingivalis } \\
\text { P. intermedia }\end{array}$ & $\begin{array}{l}\text { Probiotic use can improve } \\
\text { periodontal health through } \\
\text { plaque inhibition, } \\
\text { anti-inflammatory and } \\
\text { antimicrobial effects. }\end{array}$ \\
\hline $\begin{array}{c}\text { Vohra, } 2019 \\
\text { Saudi [109] Arabia }\end{array}$ & Periodontitis & 31 & 32 & L. reuteri & $\begin{array}{l}21 \text { days/ } \\
3 \text { months }\end{array}$ & Lozenge & SRP & Yes & $\mathrm{PII}^{*}, \mathrm{CAL}^{*}, \mathrm{BOP}^{*}, \mathrm{PPD}^{*}$ & $\begin{array}{l}\text { Probiotic use is not an effective } \\
\text { adjunct to SRP in chronic } \\
\text { periodontitis patients. }\end{array}$ \\
\hline $\begin{array}{l}\text { Yuki, } 2019[110] \\
\text { Japan }\end{array}$ & $\begin{array}{l}\text { Periodontal } \\
\text { disease }\end{array}$ & 12 & 11 & L. rhamnosus & $\begin{array}{l}90 \text { days/ } \\
90 \text { days }\end{array}$ & Yogurt & None & Yes & $\mathrm{GI}^{*}, \mathrm{PPD}^{*}, \mathrm{PMA}^{+}$ & $\begin{array}{l}\text { Probiotic use improved clinical } \\
\text { parameters under study. }\end{array}$ \\
\hline
\end{tabular}

Note: Indicates a statistically significant difference within the probiotic group from baseline to follow-up. + Indicates a statistically significant difference between the probiotic and depth $>4 \mathrm{~mm}$ and BOP. GBI Gingival bleeding inglated ble hygiene index simplified; PAL, Probing atthe hy PMA, Papillary-marginal Stain index; Microbiological-A. actinomycetemcomitans, Aggregatibacter actinomycetemcomitans; A. naeslundii, Actinomyces naeslundii; B. subtilis, Bacillus subtilis; B. megaterium, Bacillus megaterium; B. mesentericus, Bacillus mesentericus; B. pumulus, Bacillus pumulus; B. animalis, Bifidobacterium animalis; B. bifidum, Bifidobacterium bifidum; B. coagulans, Bacillus coagulans; B. lactis, Bifidobacterium lactis; B. longum, Bifidobacterium longum; C. rectus, Campylobacter rectus; C. butyricum, Clostridium butyricum; E. corrodens, Eikenella corrodens; E. faecium, Enterococcus faecium; F. alocis, Filifactor alocis; F. nucleatum, Fusobacterium nucleatum; L. acidophilus, Lactobacillus acidophilus; L. brevis, Lactobacillus brevis; L. bulgaricus, Lactobacillus bulgaricus; L. casei, Lactobacillus casei; L. crispatus, Lactobacillus crispatus; L. curvatus, Lactobacillus curvatus; L. delbrueckii, Lactobacillus delbrueckii; L. fermentum, Lactobacillus fermentum; L. plantarum, Lactobacillus plantarum, L. rhamnosus, Lactobacillus rhamnosus; L. reuteri, Lactobacillus reuteri; L. salivarius, Lactobacillus salivarius; L. sporogenes, Lactobacillus sporogenes; L. Lactis, Lactococcus lactis; P. acidilactici, Pediococcus acidilactici; P. endodontalis, Porphyromonas endodontalis; P. gingivalis, Porphyromonas gingivalis; P. intermedia, Prevotella intermedia; P. micra, Parvimonas micra; S. faecalis, Streptococcus faecalis; S. intermedia, Streptococcus intermedia; S. mutans, Streptococcus mutans; S. oralis, Streptococcus oralis; S. rattus, Streptococcus rattus; $S$. salivarius, Streptococcus salizarius; S. sanguinis, Strepto S. in Strept V. denticol $V$.

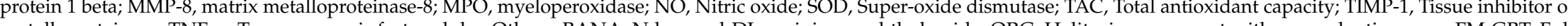
metalloproteinase; TNF- $\alpha$, Tumor necrosis factor alpha; Other-BANA, N-benzoyl-DL-arginine-naphthylamide; ORG, Halitosis assessment with organoleptic scores; FM-GBT, Ful mouth guided biofilm therapy; IPT, Initial periodontal therapy; NSPT, Non-surgical periodontal therapy; PMPR, Professional manual plaque removal; RSD, Root surface debridement, SPT, Supporting periodontal therapy; SRP, Scaling and root planing; TVC, Total viable count. 


\subsection{Risk of Bias within Studies}

Using the Cochrane risk-of-bias assessment tool version 2, the majority of studies included were classified as having a low risk of bias $(n=38)$. Additionally, 13 studies were classified as having some concerns, and another 13 studies were classified as having a high risk of bias. The majority of concerns were due to questions related to the randomization process domain. There were minimal concerns regarding the missing outcome data domain. The results are presented in Supplemental Table S3.

\subsection{Synthesis of Results}

This meta-analysis used the Hedge's g standardized mean difference (SMD) to report effect sizes. Forest plots depicting the pooled meta-analysis examining the effects of probiotic supplementation on clinical, microbiological, and immunological outcomes are presented in Figures 3-5, respectively. The overall measures of effect are summarized in Supplemental Table S4. The results of subgroup analysis are presented in Table 2 with additional information available in the Supplemental File. The forest plots for the subgroup analysis of clinical parameters are depicted in Supplemental Figure S1.

Table 2. Subgroup analysis examining the effects of probiotic supplementation on clinical outcomes.

\begin{tabular}{|c|c|c|c|c|c|c|c|c|}
\hline \multirow{2}{*}{$\begin{array}{l}\text { Clinical } \\
\text { Outcomes }\end{array}$} & \multirow{2}{*}{ Subgroup } & \multirow{2}{*}{$\begin{array}{l}\text { Level of } \\
\text { Subgroup }\end{array}$} & \multirow{2}{*}{ SMD } & \multirow{2}{*}{$95 \%$ CI } & \multirow{2}{*}{$\mathrm{I}^{2}$} & \multirow{2}{*}{$p$-Value } & \multicolumn{2}{|c|}{ Sample Size } \\
\hline & & & & & & & Probiotic & Control \\
\hline \multirow{16}{*}{$\begin{array}{l}\text { Plaque index } \\
\text { (PII) }\end{array}$} & \multirow{2}{*}{$\begin{array}{c}\text { Type of periodontal } \\
\text { disease }\end{array}$} & Gingivitis & 0.153 & $-0.152,0.457$ & 20.906 & 0.281 & 108 & 99 \\
\hline & & Periodontitis & 0.736 & $0.267,1.206$ & 71.842 & 0.001 & 136 & 135 \\
\hline & \multirow{3}{*}{$\begin{array}{c}\text { Type of probiotic } \\
\text { strain }\end{array}$} & Lactobacillus & 0.639 & $0.169,1.110$ & 75.533 & $<0.001$ & 154 & 151 \\
\hline & & Mixed & 0.280 & $-0.159,0.719$ & 0.000 & 0.523 & 42 & 36 \\
\hline & & Other & 0.185 & $-0.212,0.582$ & 0.000 & 0.431 & 48 & 47 \\
\hline & \multirow{2}{*}{$\begin{array}{c}\text { Type of Lactobacillus } \\
\text { species }\end{array}$} & L. Reuteri & 0.707 & $0.034,1.381$ & 80.976 & $<0.001$ & 98 & 95 \\
\hline & & Other & 0.590 & $-0.456,1.636$ & 81.557 & 0.004 & 42 & 42 \\
\hline & \multirow{3}{*}{ Treatment duration } & $\leq 1$ month & 0.615 & $0.146,1.084$ & 75.448 & $<0.001$ & 154 & 153 \\
\hline & & $>1$ to 2 months & 0.328 & $-0.006,0.661$ & 0.000 & 0.406 & 73 & 64 \\
\hline & & $>2$ months & 0.053 & $-0.603,0.710$ & 0.000 & 1.000 & 17 & 17 \\
\hline & \multirow{4}{*}{ Mode of delivery } & Ingestion & 0.952 & $-0.894,2.797$ & 89.037 & 0.003 & 27 & 27 \\
\hline & & Local & 0.323 & $-0.202,0.847$ & 0.000 & 1.000 & 28 & 27 \\
\hline & & Oral & 0.239 & $-0.302,0.780$ & 0.251 & 0.251 & 34 & 34 \\
\hline & & $\begin{array}{l}\text { Oral and } \\
\text { Ingestion }\end{array}$ & 0.495 & $0.061,0.930$ & 0.001 & $<0.001$ & 155 & 146 \\
\hline & \multirow{2}{*}{$\begin{array}{l}\text { Oral hygiene } \\
\text { instructions }\end{array}$} & Yes & 0.622 & $0.204,1.040$ & 66.923 & 0.006 & 145 & 138 \\
\hline & & No & 0.665 & $-0.415,1.746$ & 85.436 & $<0.001$ & 54 & 51 \\
\hline \multirow{10}{*}{$\begin{array}{l}\text { Mean plaque } \\
\text { percentage } \\
(\mathrm{MPP})\end{array}$} & \multirow{2}{*}{$\begin{array}{c}\text { Type of periodontal } \\
\text { disease }\end{array}$} & Gingivitis & 1.279 & $-0.905,3.463$ & 96.629 & $<0.001$ & 63 & 64 \\
\hline & & Periodontitis & 0.681 & $0.072,1.290$ & 82.212 & $<0.001$ & 130 & 130 \\
\hline & \multirow{3}{*}{$\begin{array}{l}\text { Type of probiotic } \\
\text { strain }\end{array}$} & Lactobacillus & 1.037 & $0.391,1.683$ & 88.278 & $<0.001$ & 195 & 198 \\
\hline & & Mixed & 0.112 & $-0.728,0.952$ & 0.000 & 1.000 & 10 & 10 \\
\hline & & Other & 0.199 & $-0.396,0.794$ & 0.000 & 1.000 & 21 & 21 \\
\hline & \multirow{2}{*}{$\begin{array}{c}\text { Type of Lactobacillus } \\
\text { species }\end{array}$} & L. Reuteri & 1.458 & $0.724,2.191$ & 86.723 & $<0.001$ & 148 & 150 \\
\hline & & Other & 0.193 & $-0.200,0.586$ & 0.000 & 0.889 & 47 & 48 \\
\hline & \multirow{3}{*}{ Treatment duration } & $\leq 1$ month & 0.937 & $0.076,1.798$ & 90.960 & $<0.001$ & 145 & 146 \\
\hline & & $>1$ to 2 months & 1.560 & $1.022,2.099$ & 0.000 & 1.000 & 33 & 35 \\
\hline & & $>2$ months & 0.460 & $0.008,0.912$ & 21.718 & 0.279 & 48 & 48 \\
\hline
\end{tabular}


Table 2. Cont.

\begin{tabular}{|c|c|c|c|c|c|c|c|c|}
\hline \multirow{2}{*}{$\begin{array}{l}\text { Clinical } \\
\text { Outcomes }\end{array}$} & \multirow{2}{*}{ Subgroup } & \multirow{2}{*}{$\begin{array}{l}\text { Level of } \\
\text { Subgroup }\end{array}$} & \multirow{2}{*}{ SMD } & \multirow{2}{*}{$95 \%$ CI } & \multirow{2}{*}{$\mathrm{I}^{2}$} & \multirow{2}{*}{$p$-Value } & \multicolumn{2}{|c|}{ Sample Size } \\
\hline & & & & & & & Probiotic & Control \\
\hline & \multirow{3}{*}{ Mode of delivery } & Ingestion & 0.969 & $-0.506,2.445$ & 94.211 & $<0.001$ & 77 & 78 \\
\hline & & Oral & 0.537 & $-0.348,1.423$ & 59.024 & 0.118 & 24 & 24 \\
\hline & & $\begin{array}{l}\text { Oral and } \\
\text { Ingestion }\end{array}$ & 0.942 & $0.159,1.725$ & 87.844 & $<0.001$ & 125 & 127 \\
\hline & \multirow{2}{*}{$\begin{array}{l}\text { Oral hygiene } \\
\text { instructions }\end{array}$} & Yes & 0.880 & $0.197,1.564$ & 88.210 & $<0.001$ & 170 & 170 \\
\hline & & No & 0.865 & $-0.502,2.232$ & 91.878 & $<0.001$ & 56 & 59 \\
\hline \multirow{16}{*}{$\begin{array}{l}\text { Gingival index } \\
\text { (GI) }\end{array}$} & \multirow{2}{*}{$\begin{array}{c}\text { Type of periodontal } \\
\text { disease }\end{array}$} & Gingivitis & 0.298 & $-0.089,0.684$ & 49.985 & 0.092 & 108 & 99 \\
\hline & & Periodontitis & 1.069 & $0.296,1.841$ & 86.299 & $<0.001$ & 116 & 112 \\
\hline & \multirow{3}{*}{$\begin{array}{l}\text { Type of probiotic } \\
\text { strain }\end{array}$} & Lactobacillus & 1.236 & $0.574,1.897$ & 87.366 & $<0.001$ & 178 & 174 \\
\hline & & Mixed & 0.101 & $-0.333,0.535$ & 0.000 & 0.949 & 43 & 36 \\
\hline & & Other & 0.329 & $-0.070,0.729$ & 0.000 & 0.354 & 48 & 47 \\
\hline & \multirow{2}{*}{$\begin{array}{l}\text { Type of Lactobacillus } \\
\text { species }\end{array}$} & L. Reuteri & 1.621 & $0.648,2.595$ & 89.871 & $<0.001$ & 112 & 111 \\
\hline & & Other & 0.817 & $0.018,1.616$ & 79.137 & 0.001 & 66 & 63 \\
\hline & \multirow{3}{*}{ Treatment duration } & $\leq 1$ month & 0.949 & $0.270,1.628$ & 85.079 & $<0.001$ & 132 & 130 \\
\hline & & $>1$ to 2 months & 0.900 & $-0.116,1.915$ & 91.498 & $<0.001$ & 106 & 99 \\
\hline & & $>2$ months & 0.888 & $-0.920,2.696$ & 89.949 & 0.002 & 31 & 28 \\
\hline & \multirow{4}{*}{ Mode of delivery } & Ingestion & 1.258 & $-0.169,2.686$ & 87.547 & $<0.001$ & 41 & 38 \\
\hline & & Local & 0.494 & $-0.035,1.023$ & 0.000 & 1.000 & 28 & 27 \\
\hline & & Oral & 0.189 & $-0.305,0.682$ & 0.000 & 0.674 & 30 & 30 \\
\hline & & $\begin{array}{l}\text { Oral and } \\
\text { Ingestion }\end{array}$ & 1.051 & $0.306,1.797$ & 89.846 & $<0.001$ & 170 & 162 \\
\hline & \multirow{2}{*}{$\begin{array}{l}\text { Oral hygiene } \\
\text { instructions }\end{array}$} & Yes & 1.051 & $0.327,1.775$ & 86.466 & $<0.001$ & 134 & 126 \\
\hline & & No & 1.344 & $0.261,2.427$ & 89.898 & $<0.001$ & 87 & 86 \\
\hline \multirow{18}{*}{$\begin{array}{l}\text { Pocket probing } \\
\text { depth (PPD) }\end{array}$} & \multirow{2}{*}{$\begin{array}{c}\text { Type of periodontal } \\
\text { disease }\end{array}$} & Gingivitis & 0.997 & $-0.853,2.848$ & 92.406 & $<0.001$ & 35 & 38 \\
\hline & & Periodontitis & 0.578 & $0.355,0.801$ & 62.720 & $<0.001$ & 442 & 434 \\
\hline & \multirow{3}{*}{$\begin{array}{l}\text { Type of probiotic } \\
\text { strain }\end{array}$} & Lactobacillus & 0.674 & $0.386,0.962$ & 69.524 & $<0.001$ & 330 & 329 \\
\hline & & Mixed & 0.387 & $0.045,0.729$ & 0.000 & 0.740 & 67 & 62 \\
\hline & & Other & 0.379 & $-0.037,0.795$ & 51.535 & 0.103 & 92 & 92 \\
\hline & \multirow{2}{*}{$\begin{array}{l}\text { Type of Lactobacillus } \\
\text { species }\end{array}$} & L. Reuteri & 0.677 & $0.315,1.040$ & 74.541 & $<0.001$ & 249 & 252 \\
\hline & & Other & 0.657 & $0.169,1.144$ & 56.911 & 0.041 & 81 & 77 \\
\hline & \multirow{3}{*}{ Treatment duration } & $\leq 1$ month & 0.737 & $0.430,1.044$ & 66.736 & $<0.001$ & 270 & 264 \\
\hline & & $>1$ to 2 months & 0.514 & $-0.030,1.059$ & 65.160 & 0.057 & 76 & 79 \\
\hline & & $>2$ months & 0.326 & $0.015,0.636$ & 43.082 & 0.080 & 143 & 140 \\
\hline & \multirow{4}{*}{ Mode of delivery } & Ingestion & 0.514 & $0.106,0.922$ & 47.870 & 0.088 & 94 & 91 \\
\hline & & Local & 0.919 & $0.370,1.468$ & 0.000 & 1.000 & 28 & 27 \\
\hline & & Oral & 0.918 & $-0.071,1.907$ & 79.291 & 0.008 & 44 & 44 \\
\hline & & $\begin{array}{l}\text { Oral and } \\
\text { Ingestion }\end{array}$ & 0.525 & $0.251,0.800$ & 66.577 & $<0.001$ & 323 & 321 \\
\hline & \multirow{2}{*}{$\begin{array}{l}\text { Oral hygiene } \\
\text { instructions }\end{array}$} & Yes & 0.592 & $0.343,0.841$ & 63.935 & $<0.001$ & 366 & 360 \\
\hline & & No & 0.953 & $0.308,1.597$ & 65.707 & 0.054 & 66 & 69 \\
\hline & 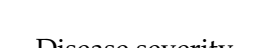 & Deep & 0.735 & $0.209,1.261$ & 73.585 & 0.002 & 112 & 114 \\
\hline & Disease severny & Moderate & 0.499 & $0.043,0.955$ & 66.202 & 0.011 & 112 & 114 \\
\hline
\end{tabular}


Table 2. Cont.

\begin{tabular}{|c|c|c|c|c|c|c|c|c|}
\hline \multirow{2}{*}{$\begin{array}{l}\text { Clinical } \\
\text { Outcomes }\end{array}$} & \multirow{2}{*}{ Subgroup } & \multirow{2}{*}{$\begin{array}{l}\text { Level of } \\
\text { Subgroup }\end{array}$} & \multirow{2}{*}{ SMD } & \multirow{2}{*}{$95 \%$ CI } & \multirow{2}{*}{$\mathbf{I}^{2}$} & \multirow{2}{*}{$p$-Value } & \multicolumn{2}{|c|}{ Sample Size } \\
\hline & & & & & & & Probiotic & Control \\
\hline \multirow{16}{*}{$\begin{array}{l}\text { Clinical } \\
\text { attachment } \\
\text { level (CAL) }\end{array}$} & \multirow{3}{*}{$\begin{array}{c}\text { Type of probiotic } \\
\text { strain }\end{array}$} & Lactobacillus & 0.417 & $0.225,0.609$ & 8.881 & 0.355 & 229 & 228 \\
\hline & & Mixed & 0.395 & $-0.066,0.855$ & 0.000 & 0.401 & 38 & 34 \\
\hline & & Other & 0.415 & $0.076,0.755$ & 7.610 & 0.339 & 72 & 72 \\
\hline & \multirow{2}{*}{$\begin{array}{c}\text { Type of Lactobacillus } \\
\text { species }\end{array}$} & L. Reuteri & 0.416 & $0.201,0.631$ & 12.027 & 0.330 & 189 & 189 \\
\hline & & Other & 0.445 & $-0.086,0.975$ & 31.016 & 0.235 & 40 & 39 \\
\hline & \multirow{3}{*}{ Treatment duration } & $\leq 1$ month & 0.388 & $0.185,0.592$ & 0.000 & 0.547 & 186 & 181 \\
\hline & & $>1$ to 2 months & 0.789 & $0.236,1.343$ & 34.507 & 0.217 & 41 & 41 \\
\hline & & $>2$ months & 0.330 & $0.071,0.588$ & 0.000 & 0.571 & 112 & 112 \\
\hline & \multirow{4}{*}{ Mode of delivery } & Ingestion & 0.464 & $0.116,0.812$ & 0.276 & 0.390 & 63 & 63 \\
\hline & & Local & 0.696 & $0.159,1.233$ & 0.000 & 1.000 & 28 & 27 \\
\hline & & Oral & 0.887 & $0.132,1.643$ & 0.000 & 1.000 & 14 & 14 \\
\hline & & $\begin{array}{l}\text { Oral and } \\
\text { Ingestion }\end{array}$ & 0.339 & $0.159,0.520$ & 0.000 & 0.543 & 234 & 230 \\
\hline & \multirow{2}{*}{$\begin{array}{l}\text { Oral hygiene } \\
\text { instructions }\end{array}$} & Yes & 0.351 & $0.178,0.523$ & 0.000 & 0.789 & 256 & 251 \\
\hline & & No & 0.835 & $0.437,1.233$ & 0.000 & 0.376 & 51 & 51 \\
\hline & \multirow{2}{*}{ Disease severity } & Deep & 0.373 & $0.088,0.657$ & 0.000 & 0.690 & 92 & 94 \\
\hline & & Moderate & 0.422 & $0.137,0.706$ & 0.000 & 0.886 & 92 & 94 \\
\hline \multirow{15}{*}{$\begin{array}{c}\text { Bleeding on } \\
\text { probing (BOP) }\end{array}$} & \multirow{2}{*}{$\begin{array}{c}\text { Type of periodontal } \\
\text { disease }\end{array}$} & Gingivitis & 0.685 & $-0.438,1.808$ & 93.899 & $<0.001$ & 117 & 120 \\
\hline & & Periodontitis & 0.749 & $0.404,1.094$ & 72.526 & $<0.001$ & 260 & 257 \\
\hline & \multirow{3}{*}{$\begin{array}{c}\text { Type of probiotic } \\
\text { strain }\end{array}$} & Lactobacillus & 0.878 & $0.442,1.313$ & 85.057 & $<0.001$ & 314 & 312 \\
\hline & & Mixed & 0.035 & $-0.574,0.643$ & 0.000 & 1.000 & 19 & 21 \\
\hline & & Other & 0.202 & $-0.210,0.613$ & 0.000 & 0.640 & 44 & 44 \\
\hline & \multirow{2}{*}{$\begin{array}{c}\text { Type of Lactobacillus } \\
\text { species }\end{array}$} & L. Reuteri & 1.054 & $0.485,1.622$ & 86.818 & $<0.001$ & 217 & 217 \\
\hline & & Other & 0.502 & $-0.078,1.081$ & 74.262 & 0.002 & 97 & 95 \\
\hline & \multirow{3}{*}{ Treatment duration } & $\leq 1$ month & 1.024 & $0.454,1.595$ & 88.021 & $<0.001$ & 236 & 238 \\
\hline & & $>1$ to 2 months & 0.095 & $-0.513,0.703$ & 0.000 & 1.000 & 20 & 20 \\
\hline & & $>2$ months & 0.402 & $0.020,0.785$ & 55.314 & 0.037 & 121 & 119 \\
\hline & \multirow{3}{*}{ Mode of delivery } & Ingestion & 0.742 & $-0.391,1.876$ & 93.499 & $<0.001$ & 112 & 110 \\
\hline & & Oral & 1.166 & $-0.037,2.370$ & 89.525 & $<0.001$ & 63 & 65 \\
\hline & & $\begin{array}{l}\text { Oral and } \\
\text { Ingestion }\end{array}$ & 0.616 & $0.296,0.936$ & 60.339 & 0.005 & 202 & 202 \\
\hline & \multirow{2}{*}{$\begin{array}{l}\text { Oral hygiene } \\
\text { instructions }\end{array}$} & No & 0.054 & $-0.508,0.617$ & 0.000 & 1.000 & 23 & 24 \\
\hline & & Yes & 0.966 & $0.478,1.454$ & 86.250 & $<0.001$ & 277 & 276 \\
\hline \multirow{2}{*}{$\begin{array}{c}\text { Gingival } \\
\text { crevicular fluid } \\
\text { (GCF) }\end{array}$} & \multirow{2}{*}{$\begin{array}{c}\text { Type of periodontal } \\
\text { disease }\end{array}$} & Gingivitis & 0.626 & $0.162,1.091$ & 0.000 & 0.392 & 36 & 36 \\
\hline & & Periodontitis & 0.507 & $0.027,0.986$ & 0.000 & 0.496 & 33 & 33 \\
\hline
\end{tabular}

Subgroup analysis assessing the effects of probiotic supplementation compared to a control on clinical outcomes in periodontal diseases using a random-effects model based on the: 1 . Type of periodontal disease; 2 . Type of probiotic strain; 3. Type of Lactobacillus species; 4. Treatment duration; 5 . Mode of probiotic delivery; and 6 Oral hygiene instruction. Note: Bold indicates statistically significant findings ( $p$-value $\leq 0.05)$. SMD, Hedge's $g$ standardized mean difference. $\mathrm{I}^{2}$, Measure of heterogeneity. 


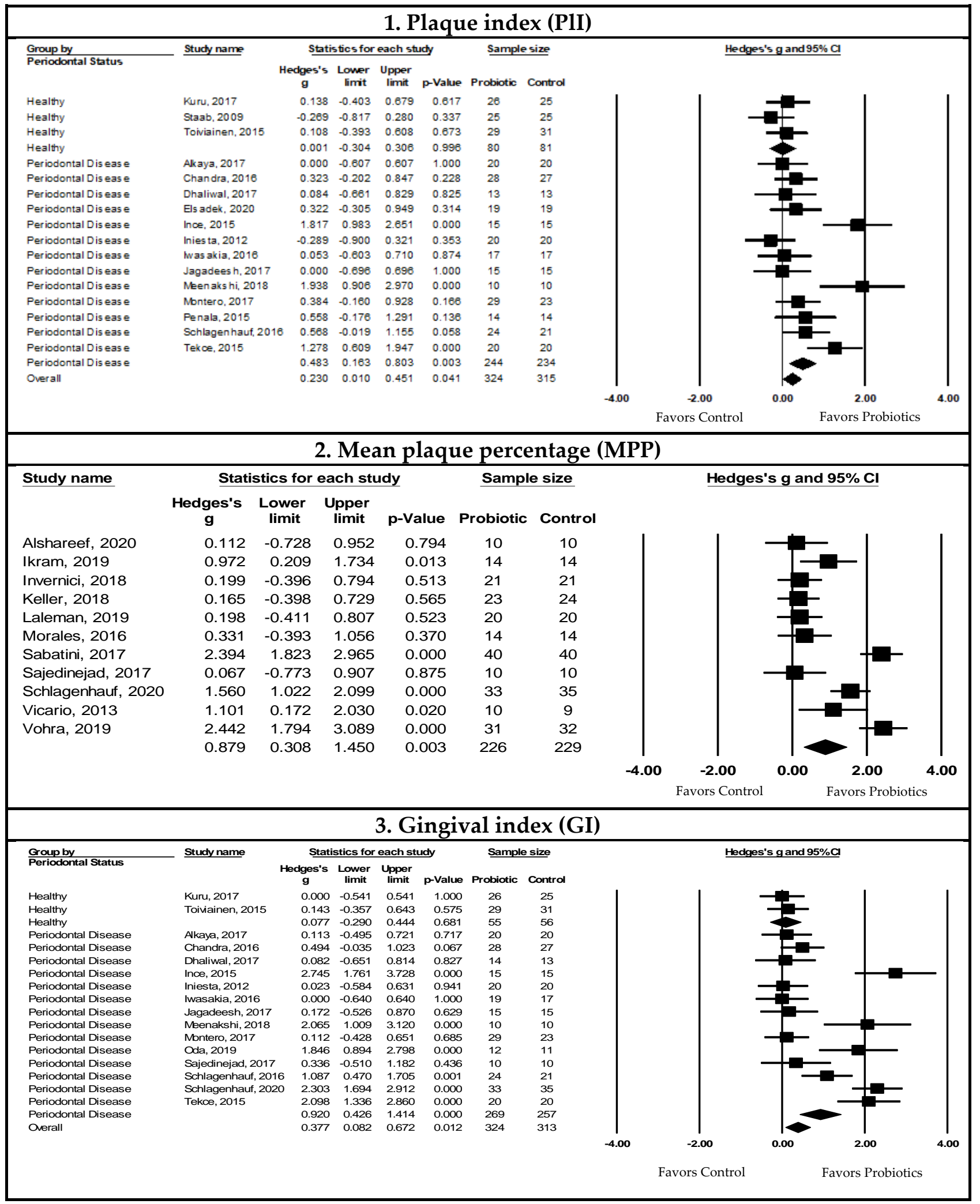

Figure 3. Cont. 


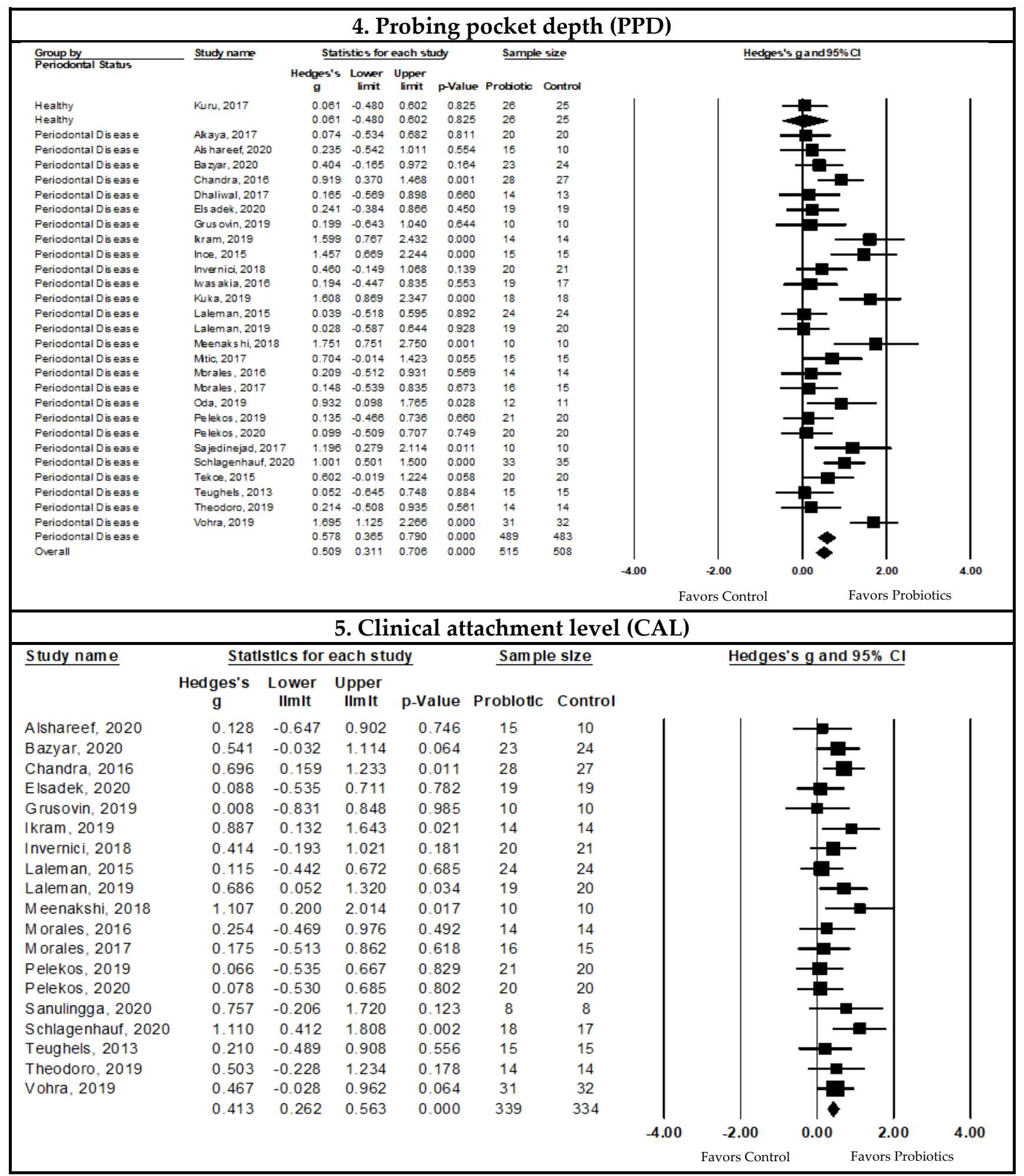

Figure 3. Cont. 


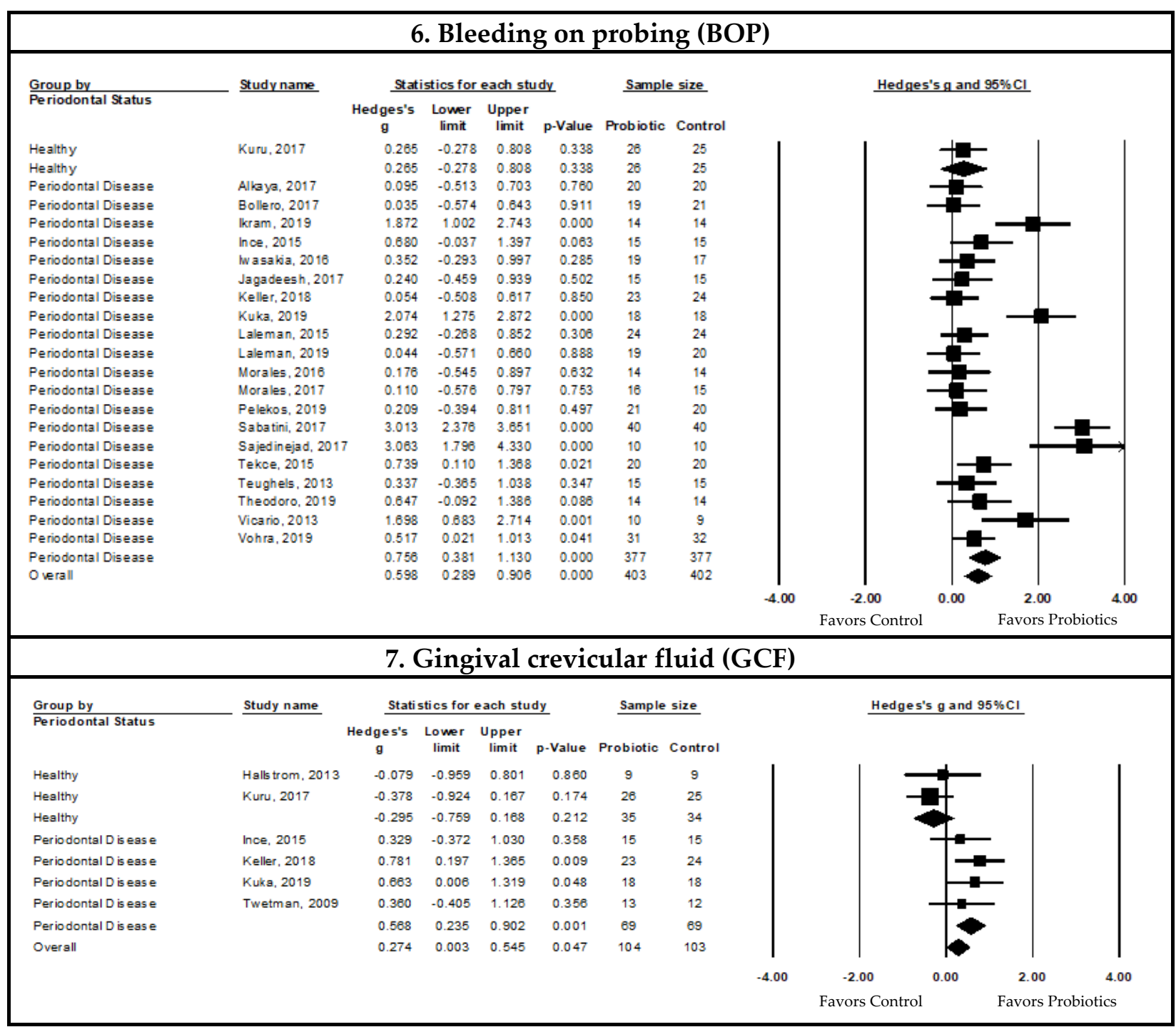

Figure 3. Pooled meta-analysis examining the effects of probiotic supplementation on clinical outcomes. 1. Forest plot of the Hedge's g SMD comparing the effects of probiotic supplementation to control groups on the plaque index (P1I) using a random-effects model. Note that we detected publication bias and/or small study effects, and the adjusted Hedge's g SMD $=0.557,95 \%$ CI: 0.228, 0.885 , and $p$-value $\leq 0.05[14,41,43,53,58,60,62,67,69,70,80,86,91,105]$. 2. Forest plot of the Hedge's $\mathrm{g}$ SMD comparing the effects of probiotic supplementation to control groups on the mean plaque percentage (MPP) using a random-effects model [54,66,68,72,77,82,88,89,92,107,109]. 3. Forest plot of the Hedge's g SMD comparing the effects of probiotic supplementation to control groups on the gingival index (GI) using a random-effects model $[14,41,43,53,58,60,69,70,75,80,89,91,92,105,110]$. 4. Forest plot of the Hedge's g SMD comparing the effects of probiotic supplementation to control groups on the probing pocket depth (PPD) using a random-effects model $[10,34,41,53$ $55,58,60,62,64,66-69,74-77,80-82,85,89,92,102,103,109,110]$. 5. Forest plot of the Hedge's g SMD comparing the effects of probiotic supplementation to control groups on the clinical attachment level (CAL) using a random-effects model [10,34,54,58,62,66,68,76,77,80,82,85,92,102,103,109]. 6. Forest plot of the Hedge's g SMD comparing the effects of probiotic supplementation to control groups on bleeding on probing (BOP) using a random-effects model. Note that we detected publication bias and/or small study effects, and the adjusted Hedge's g SMD $=0.841,95 \%$ CI: 0.479, 1.200, and $p$-value $\leq 0.05[10,34,41,53,56,66,67,69,70,72,74,76,77,88,89,102,103,107,109]$. 7. Forest plot of the Hedge's g SMD comparing the effects of probiotic supplementation to control groups on the gingival crevicular fluid (GCF) using a random-effects model [42,67,72,74,75,106]. 


\begin{tabular}{|c|c|c|c|c|c|c|c|c|c|c|}
\hline \multicolumn{11}{|c|}{ 1.P.gingivalis } \\
\hline \multirow[t]{2}{*}{ Study name } & \multicolumn{4}{|c|}{ Statistics for each study } & \multicolumn{2}{|c|}{ Sample size } & \multicolumn{4}{|c|}{ Hedges's $\mathrm{g}$ and $95 \% \mathrm{Cl}$} \\
\hline & $\begin{array}{c}\text { Hedges's } \\
\text { g }\end{array}$ & $\begin{array}{l}\text { Lower } \\
\text { limit }\end{array}$ & $\begin{array}{l}\text { Upper } \\
\text { limit }\end{array}$ & p-Value & Probiotic & Control & & & & \\
\hline Iniesta, 2012 & 0.806 & 0.174 & 1.439 & 0.012 & 20 & 20 & & & & $\mid$ \\
\hline Laleman, 2015 & 0.608 & 0.038 & 1.178 & 0.036 & 24 & 24 & & & & \\
\hline Laleman, 2019 & 0.068 & -0.547 & 0.684 & 0.828 & 19 & 20 & & & & \\
\hline Montero, 2017 & 0.127 & -0.377 & 0.631 & 0.621 & 30 & 29 & & & & \\
\hline \multirow[t]{4}{*}{ Teughels, 2013} & 0.538 & -0.172 & 1.247 & 0.137 & 15 & 15 & & & & \\
\hline & 0.402 & 0.120 & 0.685 & 0.005 & 108 & 108 & & & & \\
\hline & & & & & & & -4.00 & -2.00 & 2.00 & 4.00 \\
\hline & & & & & & & \multicolumn{3}{|c|}{ Favors Control } & \\
\hline \multicolumn{11}{|c|}{ 2. F. nucleatum } \\
\hline \multirow[t]{2}{*}{ Study name } & \multicolumn{4}{|c|}{ Statistics for each study } & \multicolumn{2}{|c|}{ Sample size } & \multicolumn{4}{|c|}{ Hedges's $\mathrm{g}$ and $95 \% \mathrm{Cl}$} \\
\hline & $\begin{array}{c}\text { Hedges's } \\
\text { g }\end{array}$ & $\begin{array}{l}\text { Lower } \\
\text { limit }\end{array}$ & $\begin{array}{c}\text { Upper } \\
\text { limit }\end{array}$ & p-Value & Probiotic & Control & & & & \\
\hline Iniesta, 2012 & 0.433 & -0.181 & 1.048 & 0.167 & 20 & 20 & & & & \\
\hline Laleman, 2015 & 0.764 & 0.187 & 1.341 & 0.009 & 24 & 24 & & & & \\
\hline Laleman, 2019 & 0.089 & -0.526 & 0.704 & 0.777 & 19 & 20 & & & & \\
\hline Montero, 2017 & 0.243 & -0.262 & 0.749 & 0.346 & 30 & 29 & & & & \\
\hline \multirow[t]{4}{*}{ Teughels, 2013} & 0.471 & -0.235 & 1.178 & 0.191 & 15 & 15 & & & & \\
\hline & 0.392 & 0.127 & 0.658 & 0.004 & 108 & 108 & & & & \\
\hline & & & & & & & -4.00 & -2.00 & 2.00 & 4.00 \\
\hline & & & & & & & \multicolumn{3}{|c|}{ Favors Control } & \\
\hline \multicolumn{11}{|c|}{ 3. T. forsythia } \\
\hline \multirow[t]{2}{*}{ Study name } & \multicolumn{4}{|c|}{ Statistics for each study } & \multicolumn{2}{|c|}{ Sample size } & \multicolumn{4}{|c|}{ Hedges's $\mathrm{g}$ and $95 \% \mathrm{Cl}$} \\
\hline & $\begin{array}{c}\text { Hedges's } \\
\text { g }\end{array}$ & $\begin{array}{l}\text { Lower } \\
\text { limit }\end{array}$ & $\begin{array}{l}\text { Upper } \\
\text { limit }\end{array}$ & p-Value & Probiotic & Control & & & & \\
\hline Iniesta, 2012 & 0.213 & -0.396 & 0.822 & 0.494 & 20 & 20 & & & & \\
\hline Laleman, 2015 & 0.541 & -0.026 & 1.108 & 0.061 & 24 & 24 & & & & \\
\hline Montero, 2017 & 0.336 & -0.171 & 0.843 & 0.194 & 30 & 29 & & & & \\
\hline \multirow[t]{4}{*}{ Teughels, 2013} & 0.217 & -0.481 & 0.916 & 0.542 & 15 & 15 & & & & \\
\hline & 0.341 & 0.050 & 0.633 & 0.022 & 89 & 88 & & & & \\
\hline & & & & & & & -4.00 & -2.00 & 0.00 & 4.00 \\
\hline & & & & & & & \multicolumn{3}{|c|}{ Favors Control } & tics \\
\hline
\end{tabular}

Figure 4. Pooled meta-analysis examining the effects of probiotic supplementation on microbiological outcomes in periodontal disease patients. 1. Forest plot of the Hedge's g SMD comparing the effects of probiotic supplementation to control groups on the subgingival $P$. gingivalis bacterial count using a random-effects model $[14,43,76,77,102]$. 2. Forest plot of the Hedge's g SMD comparing the effects of probiotic supplementation to control groups on the subgingival F. nucleatum bacterial count using a random-effects model $[14,43,76,77,102]$. 3. Forest plot of the Hedge's g SMD comparing the effects of probiotic supplementation to control groups on the subgingival T. forsythia bacterial count using a random-effects model $[14,43,76,102]$. 


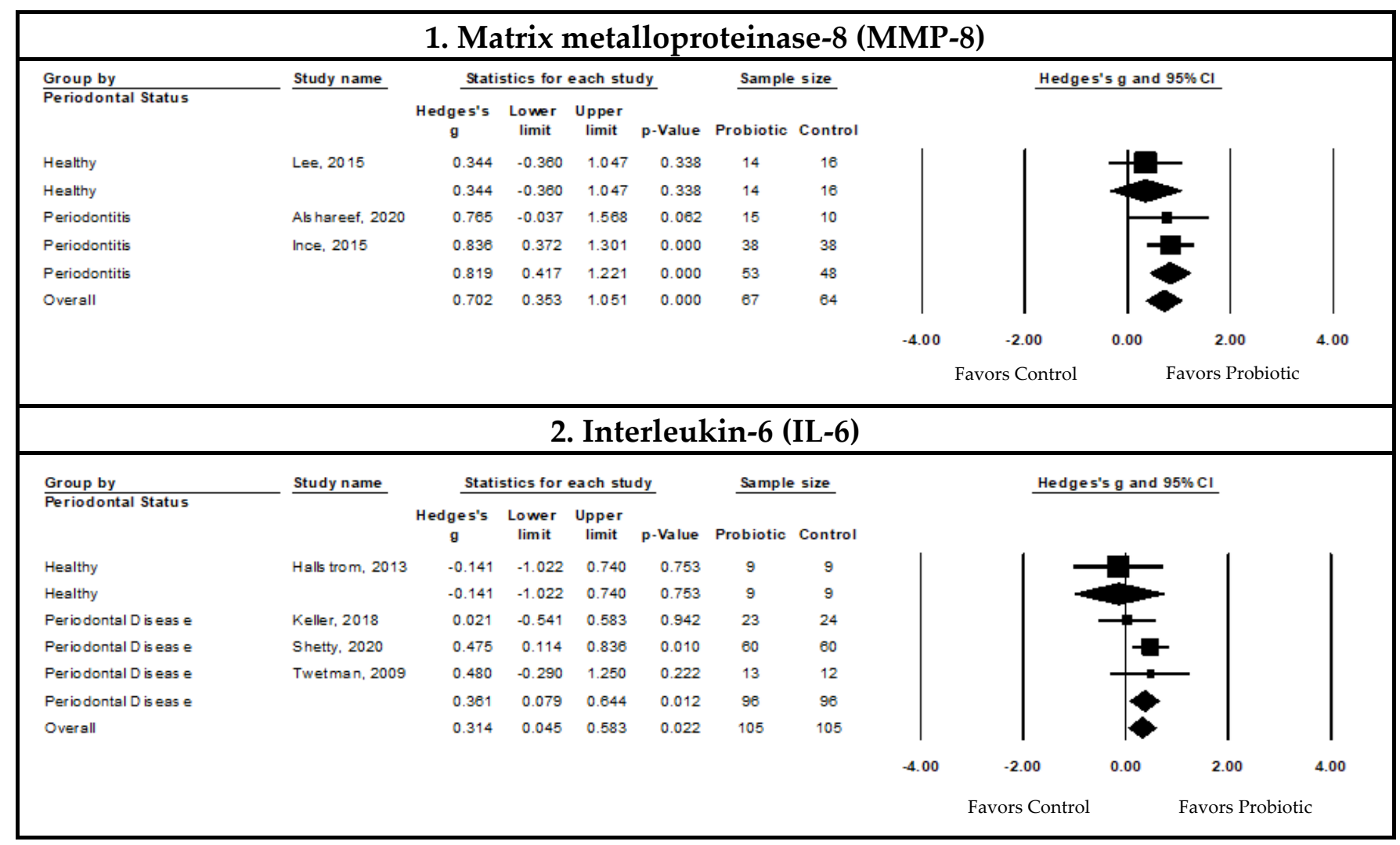

Figure 5. Pooled meta-analysis examining the effects of probiotic supplementation on immunological outcomes in periodontal disease patients; 1 . Forest plot of the Hedge's g SMD comparing the effects of probiotic supplementation to control groups on the gingival crevicular fluid (GCF) level of matrix metalloproteinase-8 (MMP-8) using a random-effects model $[59,67,78]$. 2. Forest plot of the Hedge's g SMD comparing the effects of probiotic supplementation to control groups on the GCF level of interleukin-6 (IL-6) using a random-effects model [42,72,95,106].

3.4.1. Associations between Probiotic Supplementation and Clinical Outcomes in Periodontal Disease Patients

Pooled Meta-Analysis Examining the Effects of Probiotics on Plaque Index (PII)

The effect of probiotic supplementation on the plaque index (PII) indicated a statistically significant decrease with probiotic supplementation compared to controls in patients with periodontal diseases $\left(\mathrm{SMD}=0.483,95 \% \mathrm{CI}: 0.163,0.803, \mathrm{I}^{2}=67.044, p\right.$-value $\leq 0.05$, and $n=13$ studies). Upon visual inspection of the funnel plot and confirmation through Egger's regression test ( $p$-value $=0.040)$, there was evidence of publication bias $/$ small study effects. Duval and Tweedie's trim and fill method gave an adjusted pooled estimate $\left(\mathrm{SMD}=0.557,95 \% \mathrm{CI}: 0.228,0.885, \mathrm{I}^{2}=75.716, p\right.$-value $\leq 0.05$, and $n=13$ studies) (Figure 3 ).

Pooled Meta-Analysis Examining the Effects of Probiotics on Mean Plaque Percentage (MPP)

A change in the mean plaque percentage (MPP) was reported in 11 studies. The pooled SMD showed a statistically significant decrease in the MPP with probiotic supplementation compared to controls (SMD $=0.879,95 \% \mathrm{CI}: 0.308,1.450, \mathrm{I}^{2}=87.544, p$-value $\leq 0.05$, and $n=11$ studies). There was no evidence of publication bias or small study effects upon visual inspection of the funnel plot and confirmation through Egger's regression test ( $p$-value $>0.05)$ (Figure 3$)$. 
Pooled Meta-Analysis Examining the Effects of Probiotics on Gingival Index (GI)

The pooled SMD using data from patients with periodontal disease indicated a statistically significant reduction in the gingival index (GI) with probiotic supplementation compared to controls (SMD $=0.920,95 \%$ CI: $0.426,1.414, \mathrm{I}^{2}=86.027, p$-value $\leq 0.05$, and $n=14$ studies). There was no evidence of publication bias or small study effects upon visual inspection of the funnel plot and confirmation through Egger's regression test ( $p$-value $>0.05)$ (Figure 3).

Pooled Meta-Analysis Examining the Effects of Probiotics on Probing Pocket Depth (PPD)

The pooled SMD indicated a statistically significant decrease in the probing pocket depth (PPD) with probiotic supplementation in patients with periodontal disease compared to controls (SMD $=0.578,95 \%$ CI: $0.365,0.790, \mathrm{I}^{2}=62.840, p$-value $\leq 0.05$, and $n=27$ studies). There was no evidence of publication bias or small study effect upon visual inspection of the funnel plot, and this was confirmed by Egger's regression test ( $p$-value $>0.05$ ) (Figure 3 ).

Pooled Meta-Analysis Examining the Effects of Probiotics on Clinical Attachment Level (CAL)

The pooled meta-analysis of the effect of probiotic supplementation on the clinical attachment level (CAL) was assessed in patients with periodontitis ( $n=19$ studies). Metaanalysis was not conducted to assess the effects of probiotics on CAL in healthy participants and those with gingivitis due to a lack of studies.

The pooled SMD indicates that there was a statistically significant CAL gain with probiotic supplementation compared to controls in patients with periodontitis (SMD $=0.413$, $95 \%$ CI: $0.262,0.563, \mathrm{I}^{2}<0.001, p$-value $\leq 0.05$, and $n=19$ studies). There was no evidence of publication bias and small study effects upon visual inspection of the funnel plot, which was confirmed by Egger's regression test ( $p$-value $>0.05$ ) (Figure 3).

Pooled Meta-Analysis Examining the Effects of Probiotics on Bleeding on Probing (BOP)

The effect of probiotic supplementation on bleeding on probing (BOP) indicated a statistically significant decrease with probiotic supplementation compared to controls in patients with periodontal disease (SMD $=0.756,95 \%$ CI: 0.381, 1.130, $\mathrm{I}^{2}=83.699$, $p$-value $\leq 0.05$, and $n=20$ studies). Inspection of the funnel plot for potential publication bias or small study effects suggested a right skew. This was confirmed by Egger's regression test ( $p$-value $=0.020)$. After adjusting with Duval and Tweedie's trim and fill method, the adjusted SMD remained significant with high heterogeneity (SMD $=0.841$, 95\% CI: 0.479, 1.20; and $\mathrm{I}^{2}=86.750$ ) (Figure 3).

Pooled Meta-Analysis Examining the Effects of Probiotics on Gingival Crevicular Fluid (GCF)

The effect of probiotic supplementation on the gingival crevicular fluid (GCF) volume indicated a statistically significant decrease in the GCF with probiotic supplementation compared to controls in patients with periodontal disease (SMD $=0.568,95 \%$ CI: 0.235, $0.902, \mathrm{I}^{2}<0.001, p$-value $\leq 0.05$, and $n=$ four studies). There was no evidence of publication bias or small study effects upon visual inspection of the funnel plot and confirmation by Egger's regression test ( $p$-value $>0.05)$ (Figure 3).

Pooled Meta-Analysis Examining the Effects of Probiotics on Gingival Recession (REC)

There was no statistically significant evidence that probiotic supplementation improved gingival recession (REC) compared to controls in patients with periodontitis ( $p$-value $=0.741$, and $n=5$ studies). There was no evidence of publication bias or small study effects upon visual inspection of the funnel plot and confirmation by Egger's regression test $(p$-value $>0.05)$. 
3.4.2. Associations between Probiotic Supplementation and Microbiological Outcomes in Periodontal Disease Patients

Pooled Meta-Analysis Examining the Effects of Probiotics on Subgingival Porphyromonas Gingivalis Count

The pooled SMD indicated a statistically significant decrease in the subgingival P. gingivalis count with probiotic supplementation compared to controls in patients with periodontal disease $\left(\mathrm{SMD}=0.402,95 \% \mathrm{CI}: 0.120,0.685, \mathrm{I}^{2}=10.769, p\right.$-value $\leq 0.05$, and $n=$ five studies). There was no evidence of publication bias or small study effects through visual funnel plot inspection and Egger's regression test ( $p$-value $>0.05$ ) (Figure 4 ).

Pooled Meta-Analysis Examining the Effects of Probiotics on Subgingival Fusobacterium nucleatum Count

The pooled SMD using data from periodontal disease patients indicated a statistically significant decrease in the subgingival F. nucleatum count with probiotic supplementation compared to controls (SMD $=0.392,95 \%$ CI: 0.127, 0.658, $\mathrm{I}^{2}<0.001, p$-value $\leq 0.05$, and $n=5$ studies). There was no evidence of publication bias or small study effects through visual inspection of the funnel plot and Egger's regression test ( $p$-value $>0.05$ ) (Figure 4).

Pooled Meta-Analysis Examining the Effects of Probiotic on Subgingival Tannerella forsythia Count

The pooled SMD indicated a statistically significant decrease in the subgingival T. forsythia count with probiotic supplementation compared to controls in patients with periodontal disease $\left(\mathrm{SMD}=0.341,95 \% \mathrm{CI}\right.$ : 0.050, 0.633, $\mathrm{I}^{2}<0.001, p$-value $\leq 0.05$, and $n=$ four studies). There was no evidence of publication bias or small study effects upon visual inspection of the funnel plot and with Egger's regression test ( $p$-value $>0.05$ ) (Figure 4 ).

Pooled Meta-Analysis Examining the Effects of Probiotics on Subgingival Counts of Other periodonthopathogenes

The meta-analyses conducted to assess the effects of probiotic supplementation on the subgingival bacterial counts of $P$. intermedia ( $p$-value $=0.193$, and $n=$ four studies) and $A$. actinomycetemcomitans ( $p$-value $=0.164$, and $n=5$ studies) were not statistically significant when compared to controls.

3.4.3. Associations between Probiotic Supplementation and Immunological Outcomes in Periodontal Disease Patients

Pooled Meta-Analysis Examining the Effects of Probiotics on Matrix Metalloproteinase-8 (MMP-8) Levels in the Gingival Crevicular Fluid (GCF)

The pooled SMD indicated a statistically significant decrease in the GCF MMP-8 levels with probiotic supplementation compared to the controls in patients with periodontal disease (SMD $=0.819,95 \%$ CI: $0.417,1.221, \mathrm{I}^{2}<0.001, p$-value $\leq 0.05$, and $n=$ two studies) (Figure 5).

Pooled Meta-Analysis Examining the Effects of Probiotics on Interleukin-6 (IL-6) Levels in the Gingival Crevicular Fluid

The GCF Interleukin-6 (IL-6) levels showed statistically significant decreases with probiotic supplementation compared to controls in patients with periodontal disease $\left(\mathrm{SMD}=0.361,95 \% \mathrm{CI}: 0.079,0.644, \mathrm{I}^{2}<0.001, p\right.$-value $\leq 0.05$, and $n=$ three studies). There was no evidence of publication bias or small study effects through visual funnel plot inspection and Egger's regression test ( $p$-value > 0.05) (Figure 5).

Pooled Meta-Analysis Examining the Effects of Probiotics on Other Immunological Biomarkers in the Gingival Crevicular Fluid (GCF)

The meta-analyses conducted to assess the effects of probiotic supplementation on GCF levels of IL-1 $\beta$ ( $p$-value $=0.393$ and $n=$ three studies), IL-8 ( $p$-value $=0.434$ and $n=$ two studies), IL-10 ( $p$-value $=0.902$ and $n=$ two studies $)$, and TNF- $\alpha$ ( $p$-value $=0.495$ 
and $n=$ three studies) in periodontal disease patients were not statistically significant when compared to controls.

\section{Discussion}

The aim of this systematic review and meta-analysis was to examine if probiotic supplementation is associated with preventive and therapeutic benefits in terms of improvement of clinical, microbiological, and immunological outcomes in patients with periodontal disease. In summary, the findings of this analysis are promising and indicate that probiotic supplementation significantly improved clinical outcomes, decreased certain periodontopathogen counts, and reduced the levels of specific pro-inflammatory biomarkers in patients with periodontal disease.

The overall results of this meta-analysis showed statistically significant improvements in all clinical parameters (PII, MPP, GI, PPD, CAL, BOP, and GCF volume) in patients with periodontal disease after probiotic supplementation compared to control groups who did not receive probiotics. When examining the microbiological outcomes, the subgingival periodontopathogen counts of P. gingivalis, F. nucleatum, and T. forsythia showed statistically significant reductions with probiotic supplementation. Furthermore, there was a statistically significant reduction in the GCF levels of inflammatory mediators (MMP-8 and IL-6) with probiotic supplementation.

In addition to the pooled meta-analysis, the effects of probiotic supplementation on clinical outcomes were further assessed through subgroup analysis based on:

1. Type of periodontal disease, which indicated that probiotic supplementation improved clinical outcomes in patients with periodontitis, but not in those with gingivitis or healthy individuals. However, the GCF volume had statistically significant reductions in both gingivitis and periodontitis patients;

2. Probiotic formulations consisting of Lactobacillus species and, more specifically, L. reuteri were associated with statistically significant improvements in all clinical outcomes in patients with periodontal disease;

3. Probiotic treatment duration, which showed that probiotic supplementation resulted in statistically significant improvements in the clinical outcomes after one month of supplementation in periodontal disease patients;

4. Mode of probiotic delivery, which indicated that probiotic supplementation through the "oral and ingestion" mode was associated with statistically significant improvements in all clinical outcomes in periodontal disease patients.

5. Oral hygiene instructions along with probiotic supplementation improved PII and BOP, while GI, PPD, and CAL improved with probiotic supplementation, irrespective of the presence or absence of oral hygiene instructions.

Periodontal disease stability refers to a state of successful treatment where clinical indicators do not progress in severity and is characterized by a minimized BOP score and improved PPD and CAL scores [111,112]. The results of our meta-analysis suggest that probiotic supplementation is a promising adjuvant to the current standard of care (SRP) to improve prognosis and clinical outcomes in patients with periodontitis. Given the improvements in the clinical, immunological, and microbiological outcomes in periodontal disease patients, it seems plausible that probiotics may contribute to reaching periodontal stability.

\subsection{Probiotics and Severity of Periodontal Disease}

Our subgroup analysis did not have significant findings in gingivitis patients, which may be partially attributed to: 1 . Gingivitis being an early stage of periodontal disease, and, therefore, the clinical aspects of disease and consequently the effects of probiotic supplementation may be less notable; 2 . Some studies on gingivitis did not use probiotics along with the standard of care; and 3. These results could be due to the lack of eligible studies and the moderate to high levels of heterogeneity in our analysis. However, the statistically significant improvements that our meta-analysis found in all clinical parameters at the 
late/severe disease stages (periodontitis) adds evidence to the existing literature on the clinical benefits of probiotic supplementation in the management of periodontal disease.

\subsection{Probiotics vs. Antibiotics}

The current non-surgical standard of care for periodontal disease includes SRP, which is sometimes followed by antibiotic treatment [31,33]. However, previous studies have reported that antibiotic use, particularly long-term administration, may increase the risk of oral and gut dysbiosis, antibiotic-resistant bacteria, allergic reactions, and other side effects [42]. Probiotics are a promising adjuvant/alternative approach that can be taken safely for longer periods of time. Our meta-analysis comparing probiotic supplementation to antibiotics as adjuvants to SRP did not show any statistically significant differences in CAL and PPD in periodontitis patients. (Note: The other clinical outcomes were not assessed due to insufficient studies). This finding suggests that probiotic supplementationmay be as effective as antibiotics in improving clinical outcomes. Previous studies found similar results when either one is administered as an adjuvant to SRP $[61,83]$. Furthermore, a more recent study concluded that probiotics are a more effective adjuvant with SRP than antibiotics [113]. However, there is evidence indicating that, when probiotics or antibiotics were administered independently, neither one was more efficacious than SRP alone [10,61]. Although these conclusions warrant more research, our meta-analysis indicated improved clinical outcomes, and it is plausible from a mechanistic perspective that probiotic supplementation as an adjuvant to SRP may be a safer and more effective long-term therapeutic option in the management of periodontal disease compared to antibiotics.

\subsection{Probiotic Formulation and Duration}

Our findings also indicated that probiotic formulation and the type of probiotic strain have clinical relevance. Specifically, our subgroup analysis examining the type of probiotic strain indicated that Lactobacillus probiotic formulations improved all clinical outcomes. Further analysis into the types of Lactobacillus species identified that L. reuteri-containing probiotic formulations improved all clinical outcomes. Lactobacillus species may play a role in plaque control through direct interaction via colonization resistance, which includes competition for binding sites and nutrients or antimicrobial agent production to inhibit other oral bacteria [114]. Additional evidence suggests that L. reuteri may also influence the composition of the oral microbiome and is correlated with reduced periodontopathogens, including F. nucleatum $[115,116]$. As oral microbiome dysbiosis is one of the root causes of periodontal disease, the shift induction by L. reuteri may result in improvements in periodontal disease clinical outcomes. The only study without Lactobacillus included in our meta-analysis with statistically significant reduction of PPD and CAL, but not PII, used the subgingival delivery of Saccharomyces Boulardii [58]. S. Bouldarii is a yeast probiotic that is non-colonizing [117], which likely caused nonsignificant changes in plaque accumulation [58]. However, the improvements in other clinical parameters (PPD and CAL) may be due to its role in immunomodulation [118].

Two out of eleven studies with $L$. reuteri probiotic formulations in patients with periodontitis did not result in significant reductions in plaque accumulation [62,119]. Elsadek et al. recruited periodontitis patients with type 2 diabetes, and the most immediate followup data available for our analysis was nine weeks after ending the probiotic treatment [62]. Non-significant plaque reduction could be due to the fast turnover of the oral microbiome after probiotic treatment completion [116], although a recent meta-analysis conducted to assess the long-term efficacy of probiotics found that clinical improvements persisted for up to eleven months after the completion of probiotic treatment [47]. Our subgroup analysis based on probiotic treatment duration suggested that the beneficial effects of probiotics on clinical outcomes may occur within a month. However, the optimal treatment duration cannot be inferred from our meta-analysis because there were few studies of participants with a treatment duration of more than one month to two months. 


\subsection{Probiotic Mode of Delivery}

Studies have suggested that probiotics may have different mechanisms of action to improve periodontal disease, including direct interaction with oral bacteria and/or indirect action in the oral cavity through the modulation of innate and adaptive immunity [114] and the gut-oral microbiome axis [120]. It has also been suggested that probiotics need to have enough contact time with the oral environment to become part of the biofilm and fight against pathogens through competition for binding sites, nutrients, and the production of anti-microbial substances [114,121]. Probiotics can also interact with immune cells, altering the production of inflammatory mediators, thereby modulating the immune response in the oral cavity [114]. Probiotics are also often administered to balance the gut microbiome [122]. A well-balanced gut microbiome regulates overall immune homeostasis $[123,124]$. The high count of periodontopathogens in periodontal disease may translocate to the gut resulting in gut microbiome dysbiosis [120]. Thus, it is plausible that probiotic supplementation may improve periodontal disease severity, at least in part, via this indirect mechanism linking the gut and oral microbiomes. Therefore, different modes of probiotic delivery may improve clinical outcomes based on the type of administration (oral, ingestion, or a combination of both). Interestingly, our meta-analysis indicated that probiotic administration via 'ingestion' only improved signs of tissue destruction (PPD and CAL). However, all clinical outcomes showed improvements with 'oral and ingestion' probiotic administration, potentially due to the combined direct and indirect mechanisms of action.

\subsection{Probiotic Supplementation and Oral Hygiene Instructions}

Poor oral hygiene leads to periodontal disease progression, recurrence, and treatment failure [125]. Oral hygiene instructions (OHI) play a key role in all stages of periodontal disease management. Educating patients on the importance of oral hygiene practices and techniques to ensure motivation to adhere to oral hygiene routines are critical components of OHI [125]. Our analysis showed that, irrespective of the oral hygiene instructions, probiotic supplementation improved GI, PPD, and CAL, but not plaque accumulation. Poor plaque control is a key determinant of periodontal disease [126]. Effective OHI can control and reduce plaque accumulation and improve the gingival health status, independent of professional prophylactic care $[127,128]$. Supporting this evidence, our findings indicated that probiotic supplementation combined with $\mathrm{OHI}$ resulted in statistically significant improvements in all clinical outcomes, including plaque accumulation. This suggests that probiotics may synergistically improve clinical outcomes with $\mathrm{OHI}$, stressing the importance of $\mathrm{OHI}$ in effectively preventing/reducing plaque accumulation.

\subsection{Probiotic Supplementation and GCF Volume}

In the healthy gingival sulcus, the GCF flow and volume are lower compared to those of the inflamed gingival sulcus [25]. Increased GCF flow is a host defense mechanism to flush bacteria and their metabolites from the gingival sulcus [25]. Furthermore, the GCF content, including the microbial composition and concentration of inflammatory mediators, differs between periodontal disease and healthy individuals, as well as during disease progression and during and after treatment [129]. This is in line with findings from a study by Teles et al., who found a greater GCF volume and inflammatory mediators when comparing clinically healthy oral sites in periodontitis patients compared to healthy individuals. Their results suggested a higher risk of periodontal disease initiation and progression from healthy sites in patients with periodontitis [130]. Altogether, these findings indicate the potential clinical importance of the GCF volume and its level of inflammatory mediators as an early diagnostic test for periodontal disease prior to the onset of clinical manifestations. Based on our findings, the GCF volume was significantly decreased after probiotic supplementation in both gingivitis and periodontitis patients, and other clinical outcomes also improved in these patients $[67,72,74,106]$. Although our study did not find statistically significant differences between probiotic supplementation and GCF volume in healthy participants, it should be noted that each individual study included in our analysis 
reported that the GCF volume increased without probiotic supplementation, but not with probiotic supplementation [42,78]. Probiotics may potentially be of therapeutic value for the prevention and early treatment of periodontal disease. Due to the limited number of studies included in our analysis, our results need to be interpreted with caution until further studies fully ascertain the preventive role of probiotics in periodontal disease.

\subsection{Probiotic Supplementation and Microbiological Outcomes}

Our meta-analysis examined the effects of probiotic supplementation on subgingival bacterial counts in periodontal disease. Our results found statistically significant reductions in the periodontopathogens P. gingivalis and T. forsythia from the red complex and F. nucleatum from the orange complex after probiotic supplementation. Teles et al. reported that subgingival plaque samples from healthy sites in patients with periodontitis had a higher proportion of orange and red-complex bacteria compared to healthy individuals (without periodontitis), suggesting a higher risk for these sites to be colonized by periodontopathogens [130]. Hence, probiotics, by reducing periodontopathogens, may be beneficial in inhibiting the progression of periodontal diseases to healthy sites. Orange-complex bacteria create favorable conditions for colonization by red-complex bacteria, which are strictly anaerobic [131]. F. nucleatum plays a crucial role as a bridging organism between early and late colonizers, including red-complex bacteria [132]. Red-complex pathogens are higher in number in plaques found in deeper periodontal pockets and advanced lesions [20]. Although, in our meta-analysis, probiotic supplementation resulted in statistically significant improvements in PPD for both moderate and deep pockets, this improvement was more pronounced in deeper pockets. These findings and their potential ramifications may imply that probiotic supplementation has the potential to alter the relative abundance of orange and red-complex bacteria, thus reducing oral tissue destruction and leading to conditions favoring periodontal healing/stability.

\subsection{Probiotic Supplementation and Immunological Outcomes}

Our analysis of the effects of probiotic supplementation on immunological outcomes indicated that the GCF levels of MMP-8 and IL- 6 were reduced after probiotic supplementation in patients with periodontal disease. Previously, MMP-8 and IL-6 were identified as salivary biomarkers that increased in periodontitis $[133,134]$. MMP-8 is the main collagenase involved in periodontal disease, with the highest collagenolytic activity in GCF [135]. A recent meta-analysis reported significantly higher levels of salivary MMP-8 in periodontitis patients compared to healthy participants [135]. Similarly, a study found that the GCF in the healthy sites of periodontitis patients had elevated inflammatory mediators, including MMP-8, when compared to healthy individuals [130], suggesting that inflammatory mechanisms may occur before they are clinically diagnosed or symptomatic. Moreover, additional evidence indicates that higher GCF levels of MMP-8 may predict periodontal disease progression [136], and that MMP- 8 can also be measured as a grading biomarker to classify disease stage and progression in periodontitis [137].

IL-6 is an inflammatory mediator induced by pathogens and other pro-inflammatory cytokines, and has been reported to be elevated in periodontitis [138]. A recent metaanalysis concluded that the GCF levels of IL-6 are significantly increased in patients with chronic periodontitis [139]. However, there is conflicting evidence in the literature, with other studies suggesting that there is no correlation between the IL-6 levels and periodontal disease $[140,141]$. The proposed role of IL-6 in periodontal disease pathogenesis is through the stimulation of MMP production and the activation of pathways involved in inflammation [142]. Accordingly, there may be a link between the IL-6 levels and clinical parameters of periodontal disease. The pooled results of our meta-analysis showed statistically significant reductions in the GCF levels of IL-6 after probiotic supplementation, despite individual studies having differing results. Twetman et al. indicated that the use of $L$. reuteri probiotic gum reduced the GCF levels of IL-6 and numbers of sites with bleeding on probing [106]. Keller et al. did not observe any significant change in the GCF levels of IL-6 with other 
strains of Lactobacillus probiotics; however, BOP improved significantly compared to the baseline in both the probiotic and control groups [72]. Shetty et al. found a significant reduction in the GCF levels of IL-6 after non-Lactobacillus-containing probiotic supplementation in periodontitis patients compared to controls, but not clinical outcomes [95]. Further research is required to determine the potential effects of different probiotic formulations on inflammatory molecular pathways and their impact on the clinical aspects of periodontal diseases.

\subsection{Probiotic Supplementation and Periodontal Disease Prevention}

When examining the potential preventive role of probiotic supplementation in periodontal disease in healthy individuals, the results of our meta-analysis did not find any statistically significant improvements in PII and GI after probiotic supplementation. There were insufficient studies to analyze the effects of probiotic supplementation on other outcomes in healthy participants to clearly assess the preventive potential of probiotics in periodontal diseases.

\subsection{Strengths and Limitations}

This is the first study examining the influence of probiotic formulation, mode of delivery, treatment duration, and the impact of oral hygiene instructions on periodontal diseases using subgroup analysis. Another strength of this study is that it used an up-todate analysis of the literature, used a systematic approach, and examined emerging factors that may assist in the prevention, diagnosis, and treatment of periodontal diseases.

A limitation of this meta-analysis was that the number of studies examining microbiological and immunological outcomes was scarce; thus, some of the results need to be interpreted with caution. We used Hedge's g standardized mean difference to provide better accounting for small study sample sizes. A further limitation was that, due to the aggregated data, we were unable to examine the effects of probiotic supplementation on periodontal diseases stratified by risk factors, such as sex, age, smoking status, and comorbidities. We were also unable to investigate the correlation between periodontopathogens and inflammatory biomarkers before and after probiotic treatment.

\section{Conclusions}

This systematic review and meta-analysis highlights the potential therapeutic benefits of probiotic supplementation in the treatment of periodontal disease. The results indicate that probiotic supplementation improved the clinical parameters, reduced the subgingival bacterial counts of specific periodontopathogens, and reduced the GCF levels of some proinflammatory mediators in periodontal disease patients. The impact of probiotic supplementation on clinical outcomes is affected by the probiotic formulation, mode of delivery, treatment duration, and the type of periodontal disease. More research is needed to better assess the therapeutic and preventive value of probiotic supplementation in patients with gingivitis (early disease), as well as in healthy (without periodontal disease) individuals.

Supplementary Materials: The following supporting information can be downloaded at: https: / / www.mdpi.com/article/10.3390/nu14051036/s1, Supplemental Table S1. Inclusion and exclusion criteria, Supplemental Table S2. Search terms and strategy, Supplemental Table S3: Risk of bias within studies, Supplemental Table S4. Overall measure of effects of probiotic supplementation on clinical, immunological, and microbiological outcomes. Subgroup analysis of the associations between probiotic supplementation and clinical outcomes in periodontal diseases patients. Supplemental Figure S1. Subgroup analysis - forest plots examining the effects of probiotic supplementation on clinical outcomes: 1. Plaque Index (PII); 2. Mean Plaque Percentage (MPP); 3. Gingival Index (GI); 4. Probing Pocket Depth (PPD); 5. Clinical Attachment Level (CAL); 6. Bleeding on Probing (BOP); and 7. Gingival Crevicular Fluid (GCF). 
Author Contributions: Conceptualization, Z.G., R.M., S.P., P.P. and J.R.L.L.; methodology, Z.G., A.H.s. and R.M.; Data collection and data analysis, Z.G., R.M., A.H.s. and S.P., writing-original draft, Z.G., R.M. and A.H.s.; writing—review and editing, Z.G., R.M., A.H.s., J.R.L.L., J.L., P.P. and S.P.; Supervision, S.P. and J.R.L.L. All authors have read and agreed to the published version of the manuscript.

Funding: The authors would like to acknowledge the funding support from the Patient-oriented Research Leadership Grant (S.P.) supported by the Saskatchewan Centre for Patient Oriented Research (SCPOR) and the Saskatchewan Health Research Foundation (SHRF); Saskatchewan Centre for Patient Oriented Research Graduate (Z.G.; A.H.) and Postdoctoral Fellowships (R.M.); University of Saskatchewan College of Medicine CoMGRAD Graduate Student Fellowship (Z.G.); University of Saskatchewan Department of Biochemistry, Microbiology and Immunology Devolved Scholarship (Z.G.); Saskatchewan Innovation and Opportunity Scholarship (Z.G.); University of Saskatchewan College of Dentistry start-up funds (P.P.); and University of Saskatchewan Centennial Enhancement Chair in One Health Research (P.P.).

Institutional Review Board Statement: Not applicable.

Informed Consent Statement: Not applicable.

Acknowledgments: The authors would like to thank Janice Michael, MBA, CPA, CGA, Research Facilitator of the Colleges of Dentistry and Public Health at the University of Saskatchewan, for the English editing of this manuscript. Figure 1 made in BioRender.com (accessed on 19 December 2021).

Conflicts of Interest: The authors declare no conflict of interest.

\section{Abbreviations}

Plaque Index (PlI)

Gingival Index (GI)

Pocket Probing Depth (PPD)

Clinical Attachment Level (CAL)

Bleeding on Probing (BOP)

Gingival crevicular fluid (GCF)

Gingival recession (REC)
The plaque index is a clinical indicator of oral hygiene status that measures the level and rate of microbial plaque formation on the gingival margin of the tooth's surface. PII is also used to estimate the potential therapeutic or preventive roles of oral care products [24]. The gingival index is a numeric scoring system that assesses inflammation and structural changes in gingival tissue [27]. The probing depth is the distance between the gingival margin and base of the periodontal pocket, providing one of the most accurate parameters for the clinical diagnosis of periodontal disease [23]. CAL is measured between the fixed point of the cemento-enamel junction of the tooth and the base of the periodontal pocket. The CAL is one of the most accurate and widely used measures for the clinical diagnosis of periodontal disease [23]. $\mathrm{BOP}$ is a reliable indicator of gingival inflammation and refers to the bleeding of gingival tissue upon gentle probing [143]. GCF is an inflammatory exudate that permits non-invasive sampling from the oral cavity and is composed of serum, leukocytes, and structural cells from the periodontium and oral bacteria. It is found in the periodontal sulcus between the tooth and marginal gingiva. The GCF flow rate and volume is used as an indicator of gingival inflammation, which can be used to assess periodontal disease severity [25].

REC is a clinical measurement of the distance between the cemento-enamel junction and the free gingival margin measured in millimeters $(\mathrm{mm})$ during periodontal probing [26].

\section{References}

1. GBD 2016 Disease and Injury Incidence and Prevalence Collaborators. Global, regional, and national incidence, prevalence, and years lived with disability for 328 diseases and injuries for 195 countries, 1990-2016: A systematic analysis for the Global Burden of Disease Study 2016. Lancet 2017, 390, 1211-1259. [CrossRef]

2. Nazir, M.; Al-Ansari, A.; Al-Khalifa, K.; Alhareky, M.; Gaffar, B.; Almas, K. Global Prevalence of Periodontal Disease and Lack of Its Surveillance. Sci. World J. 2020, 2020, 2146160. [CrossRef] [PubMed] 
3. Seminario-Amez, M.; Lopez-Lopez, J.; Estrugo-Devesa, A.; Ayuso-Montero, R.; Jane-Salas, E. Probiotics and oral health: A systematic review. Med. Oral Patol. Oral Cir. Bucal 2017, 22, E282-E288. [CrossRef] [PubMed]

4. Gasner, N.S.; Schure, R.S. Periodontal Disease. In StatPearls [Internet]; StatPearls Publishing: Treasure Island, FL, USA, 2021.

5. Nazir, M.A. Prevalence of periodontal disease, its association with systemic diseases and prevention. Int. J. Health Sci. 2017, 11, 72-80.

6. Ferreira, M.C.; Dias-Pereira, A.C.; Branco-de-Almeida, L.S.; Martins, C.C.; Paiva, S.M. Impact of periodontal disease on quality of life: A systematic review. J. Periodontal Res. 2017, 52, 651-665. [CrossRef] [PubMed]

7. Hajishengallis, G.; Chavakis, T. Local and systemic mechanisms linking periodontal disease and inflammatory comorbidities. Nat. Rev. Immunol. 2021, 21, 426-440. [CrossRef] [PubMed]

8. Cardoso, E.M.; Reis, C.; Manzanares-Céspedes, M.C. Chronic periodontitis, inflammatory cytokines, and interrelationship with other chronic diseases. Postgrad. Med. 2018, 130, 98-104. [CrossRef]

9. Pihlstrom, B.L.; Michalowicz, B.S.; Johnson, N.W. Periodontal diseases. Lancet 2005, 366, 1809-1820. [CrossRef]

10. Morales, A.; Gandolfo, A.; Bravo, J.; Carvajal, P.; Silva, N.; Godoy, C.; Garcia-Sesnich, J.; Hoare, A.; Diaz, P.; Gamonal, J. Microbiological and clinical effects of probiotics and antibiotics on nonsurgical treatment of chronic periodontitis: A randomized placebocontrolled trial with 9-month follow-up. J. Appl. Oral Sci. 2018, 26, e20170075. [CrossRef]

11. Könönen, E.; Gursoy, M.; Gursoy, U.K. Periodontitis: A Multifaceted Disease of Tooth-Supporting Tissues. J. Clin. Med. 2019, 8, 1135. [CrossRef]

12. Williams, R.C. Periodontal Disease. N. Engl. J. Med. 1990, 322, 373-382. [CrossRef] [PubMed]

13. Laudenbach, J.M.; Simon, Z. Common Dental and Periodontal Diseases: Evaluation and Management. Med. Clin. N. Am. 2014, 98, 1239-1260. [CrossRef] [PubMed]

14. Montero, E.; Iniesta, M.; Rodrigo, M.; Marín, M.J.; Figuero, E.; Herrera, D.; Sanz, M. Clinical and microbiological effects of the adjunctive use of probiotics in the treatment of gingivitis: A randomized controlled clinical trial. J. Clin. Periodontol. 2017, 44, 708-716. [CrossRef]

15. Shi, B.; Chang, M.; Martin, J.; Mitreva, M.; Lux, R.; Klokkevold, P.; Sodergren, E.; Weinstock, G.M.; Haak, S.K.; Lia, H. Dynamic changes in the subgingival microbiome and their potential for diagnosis and prognosis of periodontitis. MBio 2015, 6, e01926-14. [CrossRef]

16. Abusleme, L.; Dupuy, A.K.; Dutzan, N.; Silva, N.; Burleson, J.A.; Strausbaugh, L.D.; Gamonal, J.; Diaz, P.I. The subgingival microbiome in health and periodontitis and its relationship with community biomass and inflammation. ISME J. 2013, 7, 1016-1025. [CrossRef]

17. Griffen, A.L.; Beall, C.J.; Campbell, J.H.; Firestone, N.D.; Kumar, P.S.; Yang, Z.K.; Podar, M.; Leys, E.J. Distinct and complex bacterial profiles in human periodontitis and health revealed by $16 \mathrm{~S}$ pyrosequencing. ISME J. 2012, 6, 1176-1185. [CrossRef] [PubMed]

18. Liu, B.; Faller, L.L.; Klitgord, N.; Mazumdar, V.; Ghodsi, M.; Sommer, D.D.; Gibbons, T.R.; Treangen, T.J.; Chang, Y.C.; Li, S.; et al. Deep sequencing of the oral microbiome reveals signatures of periodontal disease. PLoS ONE 2012, 7, e37919. [CrossRef]

19. Curtis, M.A.; Diaz, P.I.; Van Dyke, T.E. The role of the microbiota in periodontal disease. Periodontol. 2000 2020, 83, 14-25. [CrossRef]

20. Mohanty, R.; Asopa, S.J.; Joseph, M.D.; Singh, B.; Rajguru, J.P.; Saidath, K.; Sharma, U. Red complex: Polymicrobial conglomerate in oral flora: A review. J. Fam. Med. Prim. Care 2019, 8, 3480-3486. [CrossRef]

21. Kinane, D.F.; Stathopoulou, P.G.; Papapanou, P.N. Periodontal diseases. Nat. Rev. Dis. Primers 2017, 3, 17038. [CrossRef]

22. Lamont, R.J.; Koo, H.; Hajishengallis, G. The oral microbiota: Dynamic communities and host interactions. Nat. Rev. Microbiol. 2018, 16, 745-759. [CrossRef] [PubMed]

23. Armitage, G.C. The complete periodontal examination. Periodontol. 2000 2004, 34, 22-33. [CrossRef] [PubMed]

24. Joiner, A.C. 4-The Cleaning of Teeth. In Handbook for Cleaning/Decontamination of Surfaces; Johansson, I., Somasundaran, P., Eds.; Elsevier Science B.V.: Amsterdam, The Netherlands, 2007; pp. 371-405, ISBN 978-0-444-51664-0.

25. Subbarao, K.C.; Nattuthurai, G.S.; Sundararajan, S.K.; Sujith, I.; Joseph, J.; Syedshah, Y.P. Gingival Crevicular Fluid: An Overview. J. Pharm. Bioallied Sci. 2019, 11, S135-S139. [CrossRef] [PubMed]

26. Handelman, C.S.; Eltink, A.P.; BeGole, E. Quantitative measures of gingival recession and the influence of gender, race, and attrition. Prog. Orthod. 2018, 19, 5. [CrossRef] [PubMed]

27. Rebelo, M.A.B.; de Queiroz, A.C. Gingival Indices: State of Art. In Gingival Diseases—Their Aetiology, Prevention and Treatment; Panagakos, F.S., Davies, R.M., Eds.; IntechOpen: Rijeka, Croatia, 2011; Chapter 3.

28. Sweeting, L.A.; Davis, K.; Cobb, C.M. Periodontal Treatment Protocol (PTP) for the general dental practice. J. Dent. Hyg. JDH 2008, 82 (Suppl. 3), 16-26. [PubMed]

29. Tonetti, M.S.; Greenwell, H.; Kornman, K.S. Staging and grading of periodontitis: Framework and proposal of a new classification and case definition. J. Clin. Periodontol. 2018, 45, S149-S161. [CrossRef]

30. Tonetti, M.S.; Sanz, M. Implementation of the new classification of periodontal diseases: Decision-making algorithms for clinical practice and education. J. Clin. Periodontol. 2019, 46, 398-405. [CrossRef]

31. Comprehensive Periodontal Therapy: A Statement by the American Academy of Periodontology. J. Periodontol. 2011, 82, 943-949. [CrossRef]

32. Mirbod, S.; Matthews, D.C.; Ellis, L.; Marquez, I.C. Point of Care. JCDA 2007, 73, 137-147. 
33. Hung, H.-C.; Douglass, C.W. Meta-Analysis of the effect of scaling and root planing, surgical treatment and antibiotic therapies on periodontal probing depth and attachment loss. J. Clin. Periodontol. 2002, 29, 975-986. [CrossRef]

34. Pelekos, G.; Ho, S.N.; Acharya, A.; Leung, W.K.; McGrath, C. A double-blind, paralleled-arm, placebo-controlled and randomized clinical trial of the effectiveness of probiotics as an adjunct in periodontal care. J. Clin. Periodontol. 2019, 46, 1217-1227. [CrossRef]

35. Saini, K.; Minj, J. Multifunctional Aspects of Probiotics and Prebiotics in Health Management: An Overview BT. In Dairy Processing: Advanced Research to Applications; Minj, J., Sudhakaran, V.A., Kumari, A., Eds.; Springer: Singapore, 2020; pp. 119-131, ISBN 978-981-15-2608-4.

36. Palai, S.; Derecho, C.M.P.; Kesh, S.S.; Egbuna, C.; Onyeike, P.C. Prebiotics, Probiotics, Synbiotics and Its Importance in the Management of Diseases BT. In Functional Foods and Nutraceuticals: Bioactive Components, Formulations and Innovations; Egbuna, C., Dable Tupas, G., Eds.; Springer International Publishing: Cham, Switzerland, 2020; pp. 173-196, ISBN 978-3-030-42319-3.

37. McFarland, L.V.; Surawicz, C.M.; Greenberg, R.N.; Fekety, R.; Elmer, G.W.; Moyer, K.A.; Melcher, S.A.; Bowen, K.E.; Cox, J.L.; Noorani, Z.; et al. A Randomized Placebo-Controlled Trial of Saccharomyces boulardii in Combination with Standard Antibiotics for Clostridium difficile Disease. JAMA 1994, 271, 1913-1918. [CrossRef] [PubMed]

38. Rodgers, B.; Kirley, K.; Mounsey, A.; Ewigman, B. Prescribing an antibiotic? Pair it with probiotics. J. Fam. Pract. 2013, 62, 148-150. [PubMed]

39. Rondanelli, M.; Faliva, M.A.; Perna, S.; Giacosa, A.; Peroni, G.; Castellazzi, A.M. Using probiotics in clinical practice: Where are we now? A review of existing meta-analyses. Gut Microbes 2017, 8, 521-543. [CrossRef] [PubMed]

40. Çaglar, E.; Cildir, S.K.; Ergeneli, S.; Sandalli, N.; Twetman, S. Salivary mutans streptococci and lactobacilli levels after ingestion of the probiotic bacterium Lactobacillus reuteri ATCC 55730 by straws or tablets. Acta Odontol. Scand. 2006, 64, 314-318. [CrossRef]

41. Tekce, M.; Ince, G.; Gursoy, H.; Ipci, S.D.; Cakar, G.; Kadir, T.; Y1lmaz, S. Clinical and microbiological effects of probiotic lozenges in the treatment of chronic periodontitis: A 1-year follow-up study. J. Clin. Periodontol. 2015, 42, 363-372. [CrossRef]

42. Hallström, H.; Lindgren, S.; Yucel-Lindberg, T.T.; Dahlén, G.; Renvert, S.; Twetman, S. Effect of probiotic lozenges on inflammatory reactions and oral biofilm during experimental gingivitis. Acta Odontol. Scand. 2013, 71, 828-833. [CrossRef]

43. Iniesta, M.; Herrera, D.; Montero, E.; Zurbriggen, M.; Matos, A.R.; Marín, M.J.; Sánchez-Beltrán, M.C.; Llama-Palacio, A.; Sanz, M. Probiotic effects of orally administered Lactobacillus reuteri-containing tablets on the subgingival and salivary microbiota in patients with gingivitis. A randomized clinical trial. J. Clin. Periodontol. 2012, 39, 736-744. [CrossRef]

44. Yanine, N.; Araya, I.; Brignardello-Petersen, R.; Carrasco-Labra, A.; Gonzalez, A.; Preciado, A.; Villanueva, J.; Sanz, M.; Martin, C. Effects of probiotics in periodontal diseases: A systematic review. Clin. Oral Investig. 2013, 17, 1627-1634. [CrossRef]

45. Akram, Z.; Shafqat, S.S.; Aati, S.; Kujan, O.; Fawzy, A. Clinical efficacy of probiotics in the treatment of gingivitis: A systematic review and meta-analysis. Aust. Dent. J. 2020, 65, 12-20. [CrossRef]

46. Vives-Soler, A.; Chimenos-Kustner, E. Effect of probiotics as a complement to non-surgical periodontal therapy in chronic periodontitis: A systematic review. Med. Oral Patol. Oral Cir. Bucal 2020, 25, e161-e167. [CrossRef] [PubMed]

47. Ho, S.N.; Acharya, A.; Sidharthan, S.; Li, K.Y.; Leung, W.K.; McGrath, C.; Pelekos, G. A Systematic Review and Meta-analysis of Clinical, Immunological, and Microbiological Shift in Periodontitis After Nonsurgical Periodontal Therapy with Adjunctive Use of Probiotics. J. Evid. Based Dent. Pract. 2020, 20, 101397. [CrossRef] [PubMed]

48. Villafuerte, K.R.V.; Martinez, C.J.H.; Nobre, A.V.V.; Maia, L.P.; Tirapelli, C. What are microbiological effects of the adjunctive use of probiotics in the treatment of periodontal diseases? A systematic review. Benef. Microbes 2021, 12, 307-319. [CrossRef] [PubMed]

49. Sterne, J.A.C.; Savović, J.; Page, M.J.; Elbers, R.G.; Blencowe, N.S.; Boutron, I.; Cates, C.J.; Cheng, H.-Y.; Corbett, M.S.; Eldridge, S.M.; et al. RoB 2: A revised tool for assessing risk of bias in randomised trials. BMJ 2019, 366, 14898. [CrossRef]

50. Faraone, S.V. Interpreting estimates of treatment effects: Implications for managed care. Pharm. Ther. 2008, 33, 700-711.

51. Higgins, J.P.T.; Thompson, S.G.; Deeks, J.J.; Altman, D.G. Measuring inconsistency in meta-analyses. Br. Med. J. 2003, 327, 557-560. [CrossRef] [PubMed]

52. Duval, S.; Tweedie, R. Trim and Fill: A Simple Funnel-Plot-Based Method of Testing and Adjusting for Publication Bias in Meta-Analysis. Biometrics 2000, 56, 455-463. [CrossRef]

53. Alkaya, B.; Laleman, I.; Keceli, S.; Ozcelik, O.; Haytac, M.C.; Teughels, W. Clinical effects of probiotics containing Bacillus species on gingivitis: A pilot randomized controlled trial. J. Periodontal Res. 2017, 52, 497-504. [CrossRef]

54. Alshareef, A.; Attia, A.; Almalki, M.; Alsharif, F.; Melibari, A.; Mirdad, B.; Azab, E.; Youssef, A.R.; Dardir, A. Effectiveness of Probiotic Lozenges in Periodontal Management of Chronic Periodontitis Patients: Clinical and Immunological Study. Eur. J. Dent. 2020, 14, 281-287. [CrossRef]

55. Bazyar, H.; Maghsoumi-Norouzabad, L.; Yarahmadi, M.; Gholinezhad, H.; Moradi, L.; Salehi, P.; Haghighi-Zadeh, M.H.; Zare Javid, A. The impacts of synbiotic supplementation on periodontal indices and biomarkers of oxidative stress in type 2 diabetes mellitus patients with chronic periodontitis under non-surgical periodontal therapy. A double-blind, placebo-controlled trial. Diabetes Metab. Syndr. Obes. Targets Ther. 2020, 13, 19-29. [CrossRef]

56. Bollero, P.; Di Renzo, L.; Franco, R.; Rampello, T.; Pujia, A.; Merra, G.; De Lorenzo, A.; Docimo, R. Effects of new probiotic mouthwash in patients with diabetes mellitus and cardiovascular diseases. Eur. Rev. Med. Pharmacol. Sci. 2017, 21, 5827-5836. [PubMed]

57. Boyeena, L.; Koduganti, R.R.; Panthula, V.R.; Jammula, S.P. Comparison of efficacy of probiotics versus tetracycline fibers as adjuvant to scaling and root planing. J. Indian Soc. Periodontol. 2019, 23, 539-544. [PubMed] 
58. Chandra, R.V.; Swathi, T.; Reddy, A.A.; Chakravarthy, Y.; Nagarajan, S.; Naveen, A. Effect of a Locally Delivered ProbioticPrebiotic Mixture as an Adjunct to Scaling and Root Planing in the Management of Chronic Periodontitis. J. Int. Acad. Periodontol. 2016, 18, 67-75. [PubMed]

59. Deshmukh, M.A.; Dodamani, A.S.; Karibasappa, G.; Khairnar, M.R.; Naik, R.G.; Jadhav, H.C. Comparative Evaluation of the Efficacy of Probiotic, Herbal and Chlorhexidine Mouthwash on Gingival Health: A Randomized Clinical Trial. J. Clin. Diagn. Res. 2017, 11, ZC13-ZC16. [CrossRef] [PubMed]

60. Dhaliwal, P.K.; Grover, V.; Malhotra, R.; Kapoor, A. Clinical and Microbiological Investigation of the Effects of Probiotics Combined with Scaling and Root Planing in the Management of Chronic Periodontitis: A Randomized, Controlled Study. J. Int. Acad. Periodontol. 2017, 19, 101-108. [PubMed]

61. Duarte, C.; Al-Yagoob, A.; Al-Ani, A. Efficacy of probiotics used as a periodontal treatment aid: A pilot study. Saudi Dent. J. 2019, 31, 143-147. [CrossRef] [PubMed]

62. Elsadek, M.F.; Ahmed, B.M.; Alkhawtani, D.M.; Zia Siddiqui, A. A comparative clinical, microbiological and glycemic analysis of photodynamic therapy and Lactobacillus reuteri in the treatment of chronic periodontitis in type- 2 diabetes mellitus patients. Photodiagn. Photodyn. Ther. 2020, 29, 101629. [CrossRef]

63. Ercan, N.; Olgun, E.; Kisa, U.; Yalim, M. Effect of synbiotics in the treatment of smokers and nonsmokers with gingivitis: Randomized controlled trial. Aust. Dent. J. 2020, 65, 210-219. [CrossRef]

64. Grusovin, M.G.; Bossini, S.; Calza, S.; Cappa, V.; Garzetti, G.; Scotti, E.; Gherlone, E.F.; Mensi, M. Clinical efficacy of Lactobacillus reuteri-containing lozenges in the supportive therapy of generalized periodontitis stage III and IV, grade C: 1-year results of a double-blind randomized placebo-controlled pilot study. Clin. Oral Investig. 2020, 24, 2015-2024. [CrossRef]

65. Ikram, S.; Hassan, N.; Baig, S.; Ansari, S.A.; Borges, K.J.J.; Raffat, M.A. Clinical Efficacy of Probiotics as An Adjunct to Scaling and Root Planning in The Treatment of Chronic Periodontitis. Ann. Abbasi Shaheed Hosp. Karachi Med. Dent. Coll. 2019, $24,31-37$.

66. Ikram, S.; Hassan, N.; Baig, S.; Borges, K.J.J.; Raffat, M.A.; Akram, Z. Effect of local probiotic (Lactobacillus reuteri) vs systemic antibiotic therapy as an adjunct to non-surgical periodontal treatment in chronic periodontitis. J. Investig. Clin. Dent. 2019, 10, e12393. [CrossRef] [PubMed]

67. İnce, G.; Gürsoy, H.; İpçi, Ş.D.; Cakar, G.; Emekli-Alturfan, E.; Yılmaz, S. Clinical and Biochemical Evaluation of Lozenges Containing Lactobacillus reuteri as an Adjunct to Non-Surgical Periodontal Therapy in Chronic Periodontitis. J. Periodontol. 2015, 86, 746-754. [CrossRef] [PubMed]

68. Invernici, M.M.; Salvador, S.L.; Silva, P.H.F.; Soares, M.S.M.; Casarin, R.; Palioto, D.B.; Souza, S.L.S.; Taba, M.; Novaes, A.B.; Furlaneto, F.A.C.; et al. Effects of Bifidobacterium probiotic on the treatment of chronic periodontitis: A randomized clinical trial. J. Clin. Periodontol. 2018, 45, 1198-1210. [CrossRef] [PubMed]

69. Iwasaki, K.; Maeda, K.; Hidaka, K.; Nemoto, K.; Hirose, Y.; Deguchi, S. Daily intake of heat-killed Lactobacillus plantarum L-137 decreases the probing depth in patients undergoing supportive periodontal therapy. Oral Health Prev. Dent. 2016, 14, 207-214. [CrossRef]

70. Jagadeesh K., M.; Shenoy, N.; Talwar, A.; Shetty, S. Clinical effect of pro-biotic containing Bacillus coagulans on plaque induced gingivitis: A randomized clinical pilot study. J. Health Allied Sci. NU. 2017, 7, 7-12. [CrossRef]

71. Jäsberg, H.; Tervahartiala, T.; Sorsa, T.; Söderling, E.; Haukioja, A. Probiotic intervention influences the salivary levels of Matrix Metalloproteinase (MMP)-9 and Tissue Inhibitor of metalloproteinases (TIMP)-1 in healthy adults. Arch. Oral Biol. 2018, 85, 58-63. [CrossRef]

72. Keller, M.K.; Brandsborg, E.; Holmstrom, K.; Twetman, S. Effect of tablets containing probiotic candidate strains on gingival inflammation and composition of the salivary microbiome: A randomised controlled trial. Benef. Microbes 2018, 9, 487-494. [CrossRef]

73. Krasse, P.; Carlsson, B.; Dahl, C.; Paulsson, A.; Nilsson, A.; Sinkiewicz, G. Decreased gum bleeding and reduced gingivitis by the probiotic Lactobacillus reuteri. Swed. Dent. J. 2006, 30, 55-60.

74. Kuka, G.I.; Gursoy, H.; Emekli-Alturfan, E.; Ustundag, U.V.; Kuru, B. Evaluation of nitric oxide levels in chronic periodontitis patients treated with initial periodontal therapy and probiotic food supplements: A double blind, randomized controlled clinical trial. Biotechnol. Biotechnol. Equip. 2019, 33, 974-979. [CrossRef]

75. Kuru, B.E.; Laleman, I.; Yalnızoğlu, T.; Kuru, L.; Teughels, W. The Influence of a Bifidobacterium animalis Probiotic on Gingival Health: A Randomized Controlled Clinical Trial. J. Periodontol. 2017, 88, 1115-1123. [CrossRef]

76. Laleman, I.; Yilmaz, E.; Ozcelik, O.; Haytac, C.; Pauwels, M.; Herrero, E.R.; Slomka, V.; Quirynen, M.; Alkaya, B.; Teughels, W. The effect of a streptococci containing probiotic in periodontal therapy: A randomized controlled trial. J. Clin. Periodontol. 2015, 42, 1032-1041. [CrossRef] [PubMed]

77. Laleman, I.; Pauwels, M.; Quirynen, M.; Teughels, W. A dual-strain Lactobacilli reuteri probiotic improves the treatment of residual pockets: A randomized controlled clinical trial. J. Clin. Periodontol. 2020, 47, 43-53. [CrossRef] [PubMed]

78. Lee, J.-K.; Kim, S.-J.; Ko, S.-H.; Ouwehand, A.C.; Ma, D.S. Modulation of the host response by probiotic Lactobacillus brevis CD2 in experimental gingivitis. Oral Dis. 2015, 21, 705-712. [CrossRef] [PubMed]

79. Mayanagi, G.; Kimura, M.; Nakaya, S.; Hirata, H.; Sakamoto, M.; Benno, Y.; Shimauchi, H. Probiotic effects of orally administered Lactobacillus salivarius WB21-containing tablets on periodontopathic bacteria: A double-blinded, placebo-controlled, randomized clinical trial. J. Clin. Periodontol. 2009, 36, 506-513. [CrossRef] 
80. Swarna Meenakshi, S.; Varghese, S. Adjunctive effect of probiotic (Lactobacillus casei Shirota) to scaling and root planing in the management of chronic periodontitis. Drug Invent. Today 2018, 10, 1381-1386.

81. Mitic, K.A. Probiotics and Oral Health Research. Res. J. Pharm. Biol. Chem. Sci. 2017, 8, 1021-1029.

82. Morales, A.; Carvajal, P.; Silva, N.; Hernandez, M.; Godoy, C.; Rodriguez, G.; Cabello, R.; Garcia-Sesnich, J.; Hoare, A.; Diaz, P.I.; et al. Clinical Effects of Lactobacillus rhamnosus in Non-Surgical Treatment of Chronic Periodontitis: A Randomized Placebo-Controlled Trial With 1-Year Follow-Up. J. Periodontol. 2016, 87, 944-952. [CrossRef]

83. Nadkerny, P.V.; Ravishankar, P.L.; Pramod, V.; Agarwal, L.A.; Bhandari, S. A comparative evaluation of the efficacy of probiotic and chlorhexidine mouthrinses on clinical inflammatory parameters of gingivitis: A randomized controlled clinical study. J. Indian Soc. Periodontol. 2015, 19, 633-639. [CrossRef]

84. De Luca, P.; Carvalho, G.; Franco, A.; Kreve, S.; Avila, G.; Dias, S. Zirconia-Reinforced Lithium Silicate Biocompatibility Polished in Different Stages-An In Vitro Study. J. Int. Dent. Med. Res. 2018, 11, 759-764.

85. Pelekos, G.; Acharya, A.; Eiji, N.; Hong, G.; Leung, W.K.; McGrath, C. Effects of adjunctive probiotic L. reuteri lozenges on S/RSD outcomes at molar sites with deep pockets. J. Clin. Periodontol. 2020, 47, 1098-1107. [CrossRef]

86. Penala, S.; Kalakonda, B.; Pathakota, K.; Jayakumar, A.; Koppolu, P.; Lakshmi, B.; Pandey, R.; Mishra, A. Efficacy of local use of probiotics as an adjunct to scaling and root planing in chronic periodontitis and halitosis: A randomized controlled trial. J. Res. Pharm. Pract. 2016, 5, 86. [CrossRef] [PubMed]

87. Pudgar, P.; Povšič, K.; Čuk, K.; Seme, K.; Petelin, M.; Gašperšič, R. Probiotic strains of Lactobacillus brevis and Lactobacillus plantarum as adjunct to non-surgical periodontal therapy: 3-month results of a randomized controlled clinical trial. Clin. Oral Investig. 2021, 25, 1411-1422. [CrossRef] [PubMed]

88. Tomasello, G.; Tralongo, P.; Amoroso, F.; Damiani, P.; Sinagra, E.; Noto, M.; Arculeo, V.M.; Zein, R.J.; Saad, W.; Jurjus, A.; et al. Dysmicrobism, Inflammatory Bowel Disease and Thyroiditis: Analysis of the Literature. J. Biol. Regul. Homeost. Agents 2015, 29, 265-272. [PubMed]

89. Sajedinejad, N.; Paknejad, M.; Houshmand, B.; Sharafi, H.; Jelodar, R.; Zahiri, H.S.; Noghabi, K.A. Lactobacillus salivarius NK02: A Potent Probiotic for Clinical Application in Mouthwash. Probiotics Antimicrob. Proteins 2018, 10, 485-495. [CrossRef]

90. Scaryia, L.; Nagarathna, D.V.; Varghese, M. Probiotics in periodontal therapy. Int. J. Pharma Bio Sci. 2015, 6, 242-250.

91. Schlagenhauf, U.; Jakob, L.; Eigenthaler, M.; Segerer, S.; Jockel-Schneider, Y.; Rehn, M. Regular consumption of Lactobacillus reuteri-containing lozenges reduces pregnancy gingivitis: An RCT. J. Clin. Periodontol. 2016, 43, 948-954. [CrossRef]

92. Schlagenhauf, U.; Rehder, J.; Gelbrich, G.; Jockel-Schneider, Y. Consumption of Lactobacillus reuteri-containing lozenges improves periodontal health in navy sailors at sea: A randomized controlled trial. J. Periodontol. 2020, 91, 1328-1338. [CrossRef]

93. Shah, M.P.; Gujjari, S.K.; Chandrasekhar, V.S. Evaluation of the effect of probiotic (inersan(R)) alone, combination of probiotic with doxycycline and doxycycline alone on aggressive periodontitis-A clinical and microbiological study. J. Clin. Diagn. Res. 2013, 7, 595-600. [CrossRef]

94. Shah, M.P.; Gujjari, S.K.; Chandrasekhar, V.S. Long-term effect of Lactobacillus brevis CD2 (Inersan((R))) and/or doxycycline in aggressive periodontitis. J. Indian Soc. Periodontol. 2017, 21, 341-343. [CrossRef]

95. Shetty, S.; Srigiri, S.K.; Sheikh, K.H. A Comparative Clinical, Microbiological and Biochemical Evaluation of Guided Periodontal Pocket Recolonisation (GPR) using Synbiotics as an Adjunct to Scaling and Root Planing in Patients with Chronic Periodontitis: A Pilot Project. Int. J. Med. Res. Health Sci. 2020, 9, 20-32. [CrossRef]

96. Shimauchi, H.; Mayanagi, G.; Nakaya, S.; Minamibuchi, M.; Ito, Y.; Yamaki, K.; Hirata, H. Improvement of periodontal condition by probiotics with Lactobacillus salivarius WB21: A randomized, double-blind, placebo-controlled study. J. Clin. Periodontol. 2008, 35, 897-905. [CrossRef] [PubMed]

97. Sinkiewicz, G.; Cronholm, S.; Ljunggren, L.; Dahlen, G.; Bratthall, G. Influence of dietary supplementation with Lactobacillus reuteri on the oral flora of healthy subjects. Swed. Dent. J. 2010, 34, 197-206.

98. Slawik, S.; Staufenbiel, I.; Schilke, R.; Nicksch, S.; Weinspach, K.; Stiesch, M.; Eberhard, J. Probiotics affect the clinical inflammatory parameters of experimental gingivitis in humans. Eur. J. Clin. Nutr. 2011, 65, 857-863. [CrossRef] [PubMed]

99. Sinulingga, R.T.N.; Soeroso, Y.; Lessang, R.; Sastradipura, D.F.S. Probiotic Lactobacillus reuteri effect's on the levels of interleukin-4 in periodontitis patients after scaling and root planing. Int. J. Appl. Pharm. 2020, 12, 66-68. [CrossRef]

100. Staab, B.; Eick, S.; Knöfler, G.; Jentsch, H. The influence of a probiotic milk drink on the development of gingivitis: A pilot study. J. Clin. Periodontol. 2009, 36, 850-856. [CrossRef]

101. Suzuki, N.; Tanabe, K.; Takeshita, T.; Yoneda, M.; Iwamoto, T.; Oshiro, S.; Yamashita, Y.; Hirofuji, T. Effects of oil drops containing Lactobacillus salivarius WB21 on periodontal health and oral microbiota producing volatile sulfur compounds. J. Breath Res. 2012, 6, 017106. [CrossRef]

102. Teughels, W.; Durukan, A.; Ozcelik, O.; Pauwels, M.; Quirynen, M.; Haytac, M.C. Clinical and microbiological effects of Lactobacillus reuteri probiotics in the treatment of chronic periodontitis: A randomized placebo-controlled study. J. Clin. Periodontol. 2013, 40, 1025-1035. [CrossRef]

103. Theodoro, L.H.; Claudio, M.M.; Nuernberg, M.A.A.; Miessi, D.M.J.; Batista, J.A.; Duque, C.; Garcia, V.G. Effects of Lactobacillus reuteri as an adjunct to the treatment of periodontitis in smokers: Randomised clinical trial. Benef. Microbes 2019, 10, 375-384. [CrossRef]

104. Tobita, K.; Watanabe, I.; Tomokiyo, M.; Saito, M. Effects of heat-treated Lactobacillus crispatus KT-11 strain consumption on improvement of oral cavity environment: A randomised double-blind clinical trial. Benef. Microbes 2018, 9, 585-592. [CrossRef] 
105. Toiviainen, A.; Jalasvuori, H.; Lahti, E.; Gursoy, U.; Salminen, S.; Fontana, M.; Flannagan, S.; Eckert, G.; Kokaras, A.; Paster, B.; et al. Impact of orally administered lozenges with Lactobacillus rhamnosus GG and Bifidobacterium animalis subsp. lactis BB-12 on the number of salivary mutans streptococci, amount of plaque, gingival inflammation and the oral microbiome in healthy adults. Clin. Oral Investig. 2015, 19, 77-83. [CrossRef]

106. Twetman, S.; Derawi, B.; Keller, M.; Ekstrand, K.; Yucel-Lindberg, T.; Stecksén-Blicks, C. Short-term effect of chewing gums containing probiotic Lactobacillus reuteri on the levels of inflammatory mediators in gingival crevicular fluid. Acta Odontol. Scand. 2009, 67, 19-24. [CrossRef]

107. Vicario, M.; Santos, A.; Violant, D.; Nart, J.; Giner, L. Clinical changes in periodontal subjects with the probiotic Lactobacillus reuteri Prodentis: A preliminary randomized clinical trial. ACTA Odontol. Scand. 2013, 71, 813-819. [CrossRef]

108. Vivekananda, M.R.; Vandana, K.L.; Bhat, K.G. Effect of the probiotic Lactobacilli reuteri (Prodentis) in the management of periodontal disease: A preliminary randomized clinical trial. J. Oral Microbiol. 2010, 2, 5344. [CrossRef]

109. Vohra, F.; Bukhari, I.A.; Sheikh, S.A.; Albaijan, R.; Naseem, M.; Hussain, M. Effectiveness of scalling and root plannng with and without adjunct probiotic therapy. J. Periodontol. 2020, 91, 1177-1185. [CrossRef] [PubMed]

110. Oda, Y.; Furutani, C.; Mizota, Y.; Wakita, A.; Mimura, S.; Kihara, T.; Ohara, M.; Okada, Y.; Okada, M.; Nikawa, H. Effect of bovine milk fermented with lactobacillus rhamnosus 18020 on periodontal disease in individuals with intellectual disability: A randomized clinical trial. J. Appl. Oral Sci. 2019, 27, 1-9. [CrossRef]

111. Corbet, E.; Smales, R. Oral diagnosis and treatment planning: Part 6. Preventive and treatment planning for periodontal disease. Br. Dent. J. 2012, 213, 277-284. [CrossRef] [PubMed]

112. Lang, N.P.; Bartold, P.M. Periodontal health. J. Periodontol. 2018, 89, S9-S16. [CrossRef]

113. Butera, A.; Gallo, S.; Maiorani, C.; Molino, D.; Chiesa, A.; Preda, C.; Esposito, F.; Scribante, A. Probiotic alternative to chlorhexidine in periodontal therapy: Evaluation of clinical and microbiological parameters. Microorganisms 2021, 9, 69. [CrossRef]

114. Allaker, R.P.; Stephen, A.S. Use of Probiotics and Oral Health. Curr. Oral Health Rep. 2017, 4, 309-318. [CrossRef] [PubMed]

115. Mu, Q.; Tavella, V.J.; Luo, X.M. Role of Lactobacillus reuteri in Human Health and Diseases. Front. Microbiol. 2018, 9, 757. [CrossRef]

116. Romani Vestman, N.; Chen, T.; Lif Holgerson, P.; Öhman, C.; Johansson, I. Oral Microbiota Shift after 12-Week Supplementation with Lactobacillus reuteri DSM 17938 and PTA 5289; A Randomized Control Trial. PLoS ONE 2015, 10, e0125812. [CrossRef]

117. Narwal, A. Probiotics in Dentistry-A Review. J. Nutr. Food Sci. 2011, 1. [CrossRef]

118. Bartlett, A.; Gullickson, R.G.; Singh, R.; Ro, S.; Omaye, S.T. The link between oral and gut microbiota in inflammatory bowel disease and a synopsis of potential salivary biomarkers. Appl. Sci. 2020, 10, 6421. [CrossRef]

119. Laleman, I.; Pauwels, M.; Quirynen, M.; Teughels, W. The usage of a lactobacilli probiotic in the non-surgical therapy of peri-implantitis: A randomized pilot study. Clin. Oral Implant. Res. 2020, 31, 84-92. [CrossRef]

120. Olsen, I.; Yamazaki, K. Can oral bacteria affect the microbiome of the gut? J. Oral Microbiol. 2019, 11, 1586422. [CrossRef]

121. Çaglar, E.; Kargul, B.; Tanboga, I. Bacteriotherapy and probiotics' role on oral health. Oral Dis. 2005, 11, 131-137. [CrossRef]

122. Kim, S.-K.; Guevarra, R.B.; Kim, Y.-T.; Kwon, J.; Kim, H.; Cho, J.H.; Kim, H.B.; Lee, J.-H. Role of Probiotics in Human Gut Microbiome-Associated Diseases. J. Microbiol. Biotechnol. 2019, 29, 1335-1340. [CrossRef]

123. Belkaid, Y.; Harrison, O.J. Homeostatic Immunity and the Microbiota. Immunity 2017, 46, 562-576. [CrossRef]

124. Wu, H.-J.; Wu, E. The role of gut microbiota in immune homeostasis and autoimmunity. Gut Microbes 2012, 3, 4-14. [CrossRef]

125. Carra, M.C.; Detzen, L.; Kitzmann, J.; Woelber, J.P.; Ramseier, C.A.; Bouchard, P. Promoting behavioural changes to improve oral hygiene in patients with periodontal diseases: A systematic review. J. Clin. Periodontol. 2020, 47 (Suppl. S2), 72-89. [CrossRef]

126. Bouchard, P.; Carra, M.C.; Boillot, A.; Mora, F.; Rangé, H. Risk factors in periodontology: A conceptual framework. J. Clin. Periodontol. 2017, 44, 125-131. [CrossRef] [PubMed]

127. Singla, S.; Gupta, P.; Lehl, G.; Talwar, M. Effects of Reinforced Oral Hygiene Instruction Program With and Without Professional Tooth Cleaning on Plaque Control and Gingival Health of Orthodontic Patients Wearing Multibracket Appliances. J. Indian Orthod. Soc. 2019, 53, 272-277. [CrossRef]

128. Newton, J.T.; Asimakopoulou, K. Managing oral hygiene as a risk factor for periodontal disease: A systematic review of psychological approaches to behaviour change for improved plaque control in periodontal management. J. Clin. Periodontol. 2015, 42, S36-S46. [CrossRef] [PubMed]

129. Barros, S.P.; Williams, R.; Offenbacher, S.; Morelli, T. Gingival crevicular fluid as a source of biomarkers for periodontitis. Periodontol. 2000 2016, 70, 53-64. [CrossRef]

130. Teles, R.; Sakellari, D.; Teles, F.; Konstantinidis, A.; Kent, R.; Socransky, S.; Haffajee, A. Relationships among gingival crevicular fluid biomarkers, clinical parameters of periodontal disease, and the subgingival microbiota. J. Periodontol. 2010, 81, 89-98. [CrossRef]

131. Bourgeois, D.; Inquimbert, C.; Ottolenghi, L.; Carrouel, F. Periodontal Pathogens as Risk Factors of Cardiovascular Diseases, Diabetes, Rheumatoid Arthritis, Cancer, and Chronic Obstructive Pulmonary Disease-Is There Cause for Consideration? Microorganisms 2019, 7, 424. [CrossRef]

132. Brennan, C.A.; Garrett, W.S. Fusobacterium nucleatum-Symbiont, opportunist and oncobacterium. Nat. Rev. Microbiol. 2019, 17, 156-166. [CrossRef]

133. Javed, F.; Ahmed, H.B.; Saeed, A.; Mehmood, A.; Bain, C. Whole Salivary Interleukin-6 and Matrix Metalloproteinase- 8 Levels in Patients with Chronic Periodontitis With and Without Prediabetes. J. Periodontol. 2014, 85, e130-e135. [CrossRef] 
134. Ebersole, J.L.; Dawson, D.R.; Morford, L.A.; Peyyala, R.; Miller, C.S.; Gonzaléz, O.A. Periodontal disease immunology: “Double indemnity" in protecting the host. Periodontol. 2000 2013, 62, 163-202. [CrossRef]

135. Zhang, L.; Li, X.; Yan, H.; Huang, L. Salivary matrix metalloproteinase (MMP)-8 as a biomarker for periodontitis: A PRISMAcompliant systematic review and meta-analysis. Medicine 2018, 97, e9642. [CrossRef]

136. Al-Majid, A.; Alassiri, S.; Rathnayake, N.; Tervahartiala, T.; Gieselmann, D.-R.; Sorsa, T. Matrix Metalloproteinase-8 as an Inflammatory and Prevention Biomarker in Periodontal and Peri-Implant Diseases. Int. J. Dent. 2018, 2018, 7891323. [CrossRef]

137. Sorsa, T.; Alassiri, S.; Grigoriadis, A.; Räisänen, I.T.; Pärnänen, P.; Nwhator, S.O.; Gieselmann, D.-R.; Sakellari, D. Active MMP-8 (aMMP-8) as a Grading and Staging Biomarker in the Periodontitis Classification. Diagnostics 2020, 10, 61. [CrossRef]

138. Pan, W.; Wang, Q.; Chen, Q. The cytokine network involved in the host immune response to periodontitis. Int. J. Oral Sci. 2019, 11, 30. [CrossRef] [PubMed]

139. Stadler, A.F.; Angst, P.D.M.; Arce, R.M.; Gomes, S.C.; Oppermann, R.V.; Susin, C. Gingival crevicular fluid levels of cytokines/chemokines in chronic periodontitis: A meta-analysis. J. Clin. Periodontol. 2016, 43, 727-745. [CrossRef] [PubMed]

140. Teles, R.P.; Likhari, V.; Socransky, S.S.; Haffajee, A.D. Salivary cytokine levels in subjects with chronic periodontitis and in periodontally healthy individuals: A cross-sectional study. J. Periodontal Res. 2009, 44, 411-417. [CrossRef]

141. Goutoudi, P.; Diza, E.; Arvanitidou, M. Effect of periodontal therapy on crevicular fluid interleukin-6 and interleukin-8 levels in chronic periodontitis. Int. J. Dent. 2012, 2012, 362905. [CrossRef]

142. Ross, J.H.; Hardy, D.C.; Schuyler, C.A.; Slate, E.H.; Mize, T.W.; Huang, Y. Expression of periodontal interleukin-6 protein is increased across patients with neither periodontal disease nor diabetes, patients with periodontal disease alone and patients with both diseases. J. Periodontal Res. 2010, 45, 688-694. [CrossRef] [PubMed]

143. Checchi, L.; Montevecchi, M.; Checchi, V.; Zappulla, F. The Relationship Between Bleeding on Probing and Subgingival Deposits. An Endoscopical Evaluation. Open Dent. J. 2009, 3, 154-160. [CrossRef] 